\title{
Novel Error Concealment Method With Adaptive Prediction to the Abrupt and Gradual Scene Changes
}

\author{
Soo-Chang Pei, Fellow, IEEE, and Yu-Zuong Chou
}

\begin{abstract}
In this paper, the impact of the scene change on the conventional error concealment method is addressed and a novel error concealment method is proposed to improve the insufficiency of conventional temporal error concealment algorithm due to the occurrence of scene change. Combining with the low complexity scene change detection algorithm using macroblock type information, the corrupt blocks resulting from bit errors are concealed either temporally or spatially depending on whether or not an abrupt scene change is found. In the case of gradual scene change, a novel error concealment method of interpolation and extrapolation is proposed to utilize the linear property of gradual scene change sequence, and effectively reduce the concealment error in comparison with the conventional algorithm. Great improvement about 3 to $5 \mathrm{~dB}$ PSNR in average and 6 to $8 \mathrm{~dB}$ in some cases is obtained with very little overhead memory and computation.
\end{abstract}

Index Terms-Error concealment, MPEG-2, scene change detection, video communication.

\section{INTRODUCTION}

$\mathbf{T}$ HE MPEG video coding standard is widely used to handle the fast growing video data. Besides reducing the storage space, the smaller amount of compressed video data also facilitates the video communication. As the rapid development of wireless communication and broadband integrated services digital network (B-ISDN), the compressed video transmission technique becomes an urgent work. Although MPEG greatly reduces the video data amount and makes video communication possible, MPEG coding scheme also produces some annoying side effects to practical application. Under the noisy channel, such as the environment of wireless communication, high bit error rate will do great harm to quality of decoded MPEG video due to the error propagation effect of the prediction coding. In addition, under the asynchronous transfer mode (ATM) environment, video data packets may be dropped or lost owing to the congestion in an excessive traffic on the network. The error block produced by loss packets propagates all the group of pictures (GOP) and probably result in unacceptable decoded results. Consequently, being aware of the error source

Manuscript received March 13, 2000; revised April 4, 2002. This work was supported by the National Science Council of Taiwan, R.O.C., under Contract NSC 91-2219-E-002-044 and by the Ministry of Education under Contract 89-E-FA06-2-4. The associate editor coordinating the review of this paper and approving it for publication was Prof. Tsuhan Chen.

The authors are with the Department of Electrical Engineering National Taiwan University, Taipei 10617, Taiwan, R.O.C. (e-mail: pei@cc.ee.ntu.edu.tw)

Digital Object Identifier 10.1109/TMM.2003.819749

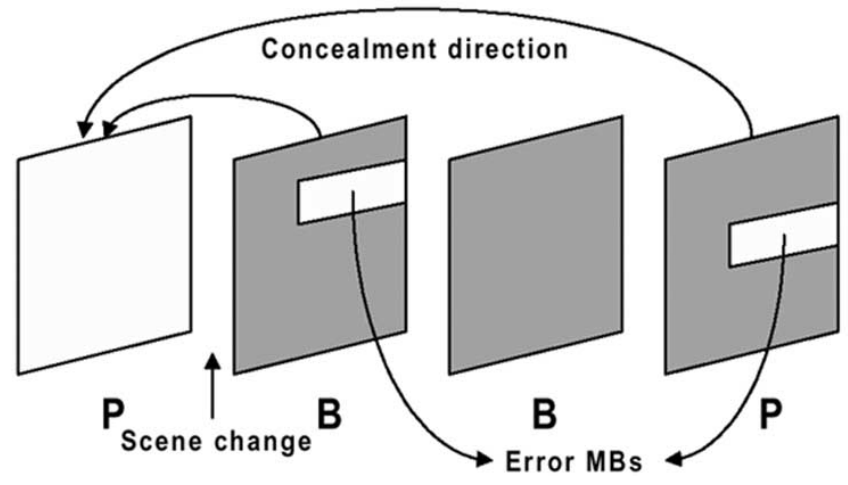

Fig. 1. Illustration of the impact of scene change on conventional temporal error concealment.

and adopting some proper action to handle the transmission errors become a very important issue. This is also the reason why more and more studies concerning error concealment [1]-[10], [13]-[26] and error resilient coding [11], [12] are proposed.

Bit error detection, which is considered as the initial step toward error concealment, can be achieved by many ways, such as the invalid codeword detection. An invalid codeword for macroblock_address_increment, macroblock_type, motion_code or DCT coefficients can be used to indicate the occurrence of the bit error. In addition, some unreasonable conditions, such as exceeding 64 coefficients existing on one block, can also be used for bit error detection. Once even only one bit error occurs, the data succeeding this error bit will be lost until the resynchronization obtained by the new-coming header of start_of_slice. The error block would propagate all the remaining frames of the GOP and greatly degrade the quality of decoded video. As a result of avoiding error propagation, several error concealment algorithms are proposed to compensate corrupt blocks. Most related researches concerning error concealment utilize the temporal correlation of neighboring frames under the assumption that neighboring frames have very similar contents. The simplest and straightforward idea, called block replacement error concealment (BREC) [8], is replacing the lost MB with the co-located MB of the previous decoded frame. The great advantage of this method is that no overhead computation and memory is needed. However, this approach only works for the pictures where there is little or no motion. It is not efficient for fast movements resulting from panning or fast moving objects. For the panning sequence like the well-known "windmill," BREC algorithm would produce annoying blocky effects resulting from visible 


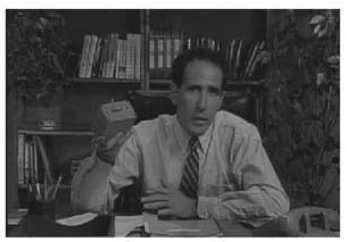

Frame 3
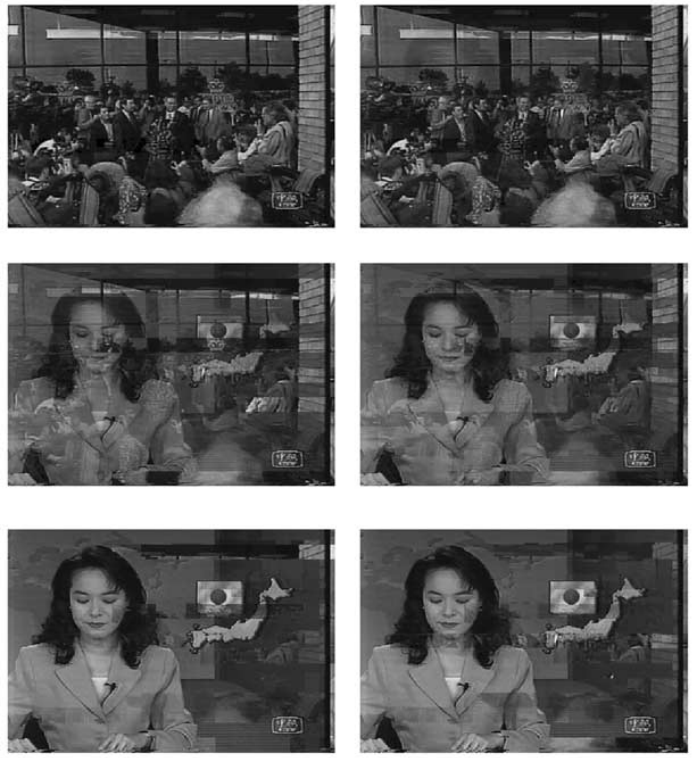

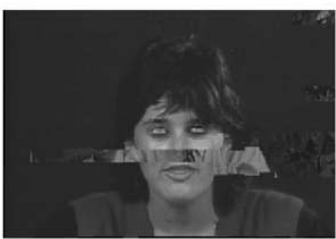

Frame 5

(a)
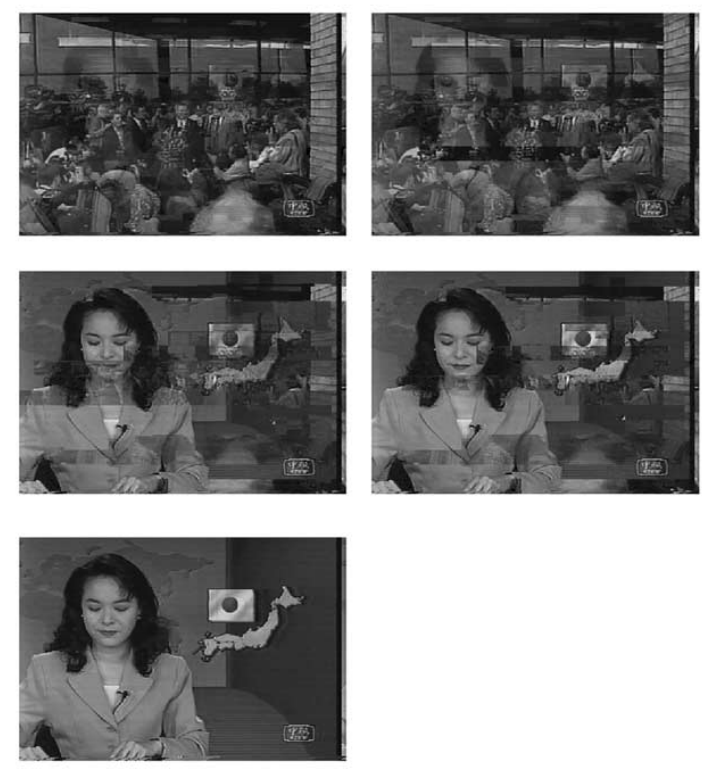

(b)

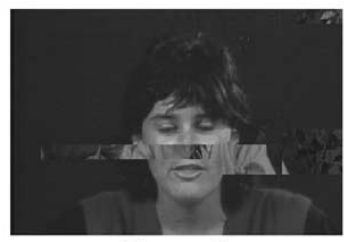

Frame 6

Fig. 2. (a) Error Concealed video sequence using temporal error concealment algorithm (scene change at Frame 4). (b) Error Concealed video sequence using temporal error concealment algorithm (news sequence spans from frame 5 to frame 15 and gradual scene change from frame 6 to frame 10).

shift. Consequently, in order to track the motion and exploit the high correlation of the motion vectors between adjacent MBs, motion-compensated error concealment method is proposed. Motion compensated temporal error concealment (MCTEC) [9] tries to estimate the motion vectors of corrupt blocks, based on which, corrupt blocks can be compensated. There are several ways to select the motion vectors of corrupt MBs. Due to the property of error propagating to the end of the slice, the most practical way is to use the motion vectors of the MB right above the lost MB. Another method of motion vector selection is the reuse of the motion estimation technique from the surrounding pixels of the lost MBs to the previous decoded frame. The motion vectors of the best matching are chosen for motion compensated error concealment. Simulation results of this method show that more accurate motion vectors are obtained and the problem resulting from different foreground and background motion is solved. Nevertheless, this re-estimated method of high computation load might delay the decoding process. Shyu and Leou [10] proposed a genetic algorithm to choose the concealed block. Concealed block based on different motion vector and reference frames are defined as different chromosome types. By iterations of crossover, mutation and reproduction, the one with best fitness is chosen as the block to conceal.

Although satisfactory error concealment results are achieved, many researches are still in progress to find the best motion vector estimation for corrupt $\mathrm{MB}$ and the best concealment policy to handle different frame types. In this paper, an unnoticed error propagation problem resulting from the occurrence of scene change is pointed out and the insufficiency of conventional algorithm to deal with the problem is discussed, hence a novel and improved error concealment strategy is proposed. Combining with the scene change detection algorithm based on MB type information, this novel algorithm lead us to make the better choice of which reference frame to predict and conceal the error, either spatial or temporal error concealment should be adopted and whether interpolation and extrapolation are needed. For the abrupt scene change, the corrupt MBs of the nearest following $P$ frame right after the scene change should be spatial error concealed because this $\mathrm{P}$ frame has no temporal correlation with the anchor P or I frame at all. Furthermore, for the B frames, the corrupt blocks should be temporal concealed with the anchor P or I frame at the same shot. By contrast, error concealment of the conventional algorithm across the scene change, i.e., the boundary between different shots, will produce very unacceptable results. In addition to the abrupt scene change, some strategies are also proposed for the gradual scene change, which are usually created by the dissolve effect. The more accurate interpolated and extrapolated error concealment algorithm can help us to reconstruct the corrupt blocks more effectively. Based on the linear property of dissolve sequence, the corrupt blocks of B frames can be concealed by 
the interpolation of two anchor frames. As well, the corrupt blocks of $\mathrm{P}$ frame can be concealed by the extrapolation of former two anchor $\mathrm{P}$ or I frames or by the interpolation of two adjacent anchor P or I frames. Certainly, the parameter of interpolation or extrapolation should be estimated in advance from the complete decoded block groups.

Simulation results show great improvement is obtained in comparison with the conventional temporal error concealment algorithm. Simulation also shows that overhead computation and extra memory space are very insignificant compared with the MPEG decoding process. Last, in order to see the impact of false alarm on our method, a simulation with false alarm insertion is tested. Only slight influence on the video quality of decoded sequence is produced.

\section{IMPACT OF THE SCENE ChANGE ON ERROR CONCEALMENT}

By using the temporal error concealment method, the corrupt MBs can be sufficiently reconstructed when dealing with ordinary video sequences. But some effects, such as scene change and dissolve, which commonly occur in the video sequences, will make conventional temporal error concealment method ineffective. Fig. 1 illustrates the impact on the error concealment method when the scene change is involved. Temporal error concealment may exploit the temporal redundancy of the frames on the two sides across the scene change to predict the corrupted MBs. Unfortunately, due to the scene change, there is no temporal redundancy at all. In other words, error concealment mechanism chooses a totally different MB of the different scene to compensate the corrupted one. Fig. 2(a) shows the insufficiency of the conventional error concealment methods. Besides, for the gradual scene change, the temporal correlation is so insignificant that motion compensation error concealment also generate very unacceptable concealment results. Fig. 2(b) shows the concealment results and indicates that conventional temporal error concealment is inadequate for the case of gradual scene change. Therefore, a novel error concealment method needs to be proposed to improve this insufficiency.

\section{Scene Change Detection Method}

For the case of scene change sequences, conventional error concealment methods are incapable to provide satisfactory results. A novel error concealment method, therefore, is introduced to solve the ineffectiveness of conventional algorithms and the error propagation problems resulting from the scene change. As the initial step, the scene change detection plays a very important role. So far, many researches concerning scene change detection have been proposed [30]-[45] and recently, as the video data grows, more and more researches focus on detecting the scene change directly from MPEG compressed video. Most of them utilize the correlation of DCT coefficients or the variance of motion vectors. Although satisfactory simulation results are obtained, a large amount of computation and memory requirement makes these methods less attractive when applying to error concealment. We have proposed an efficient scene change detection method using MB
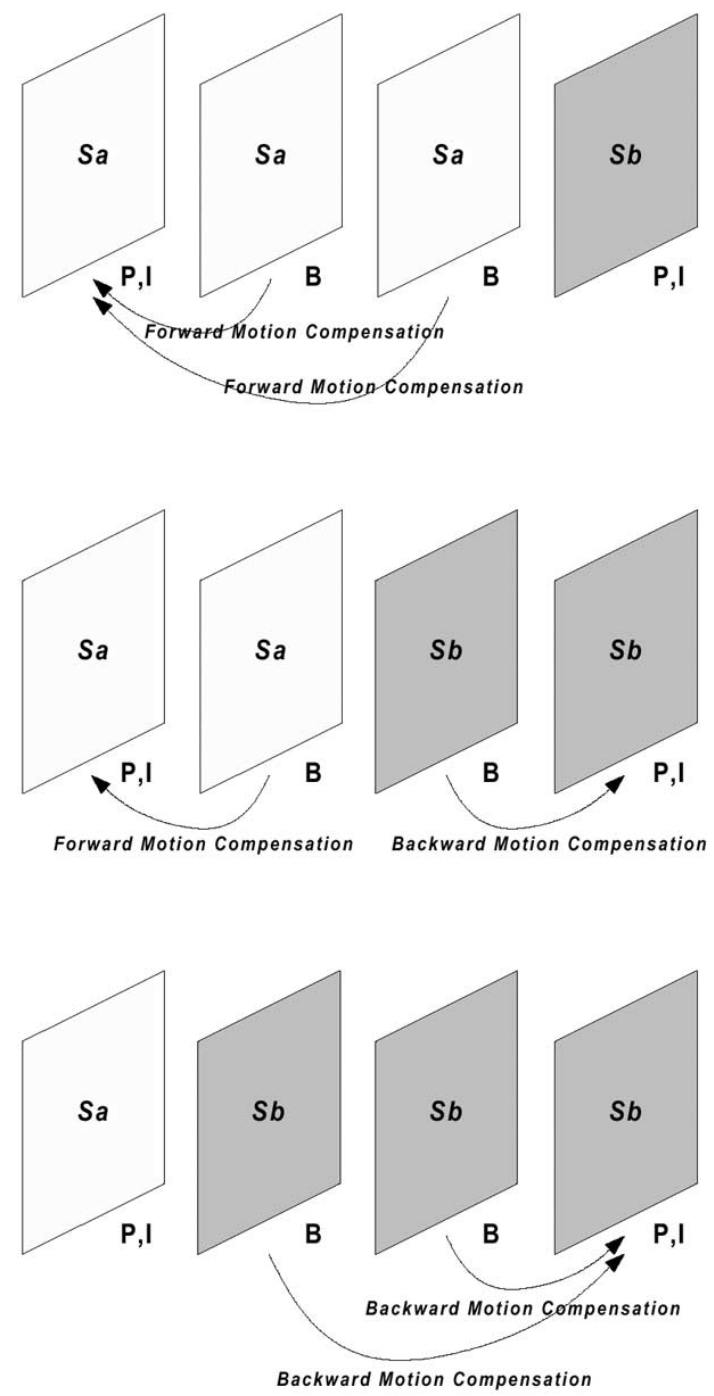

Fig. 3. Patterns of macroblock type the video sequence involving the abrupt scene change.

type information [27]. This method exploits the comparison operations performed in the motion estimation procedure, which results in specific characteristics of the MB type information when scene changes occur or some special effects occur. Figs. 3 and 4 illustrate the patterns of abrupt and gradual scene changes corresponding to different scene change occurring positions. Based on these patterns, only a simple analysis on MB types of the frames is needed to achieve very fast scene change and gradual transition detection. The advantage of low complexity and less memory requirement benefits this novel error concealment algorithm. In addition, this detection method is also attractive for its capability to handle simultaneously both abrupt scene change and gradual scene change detection. Nevertheless, it is necessary to note that the abrupt scene change detection completes only when all four frames of a SubGroup Of Pictures (SGOP), a set of PBBP, IBBP, or PBBI, which is represented as $P_{f} B_{f} B_{r} P_{r}$ (Pfront, Bfront, Brear, Prear), $I B_{f} B_{r} P_{r}$ and $P_{f} B_{f} B_{r} I$ shown in Fig. 5, are processed. This unfavorable property will increase the memory requirement and lengthen the display delay. The first B frame of a SGOP could not display immediately after decoding because it has to 

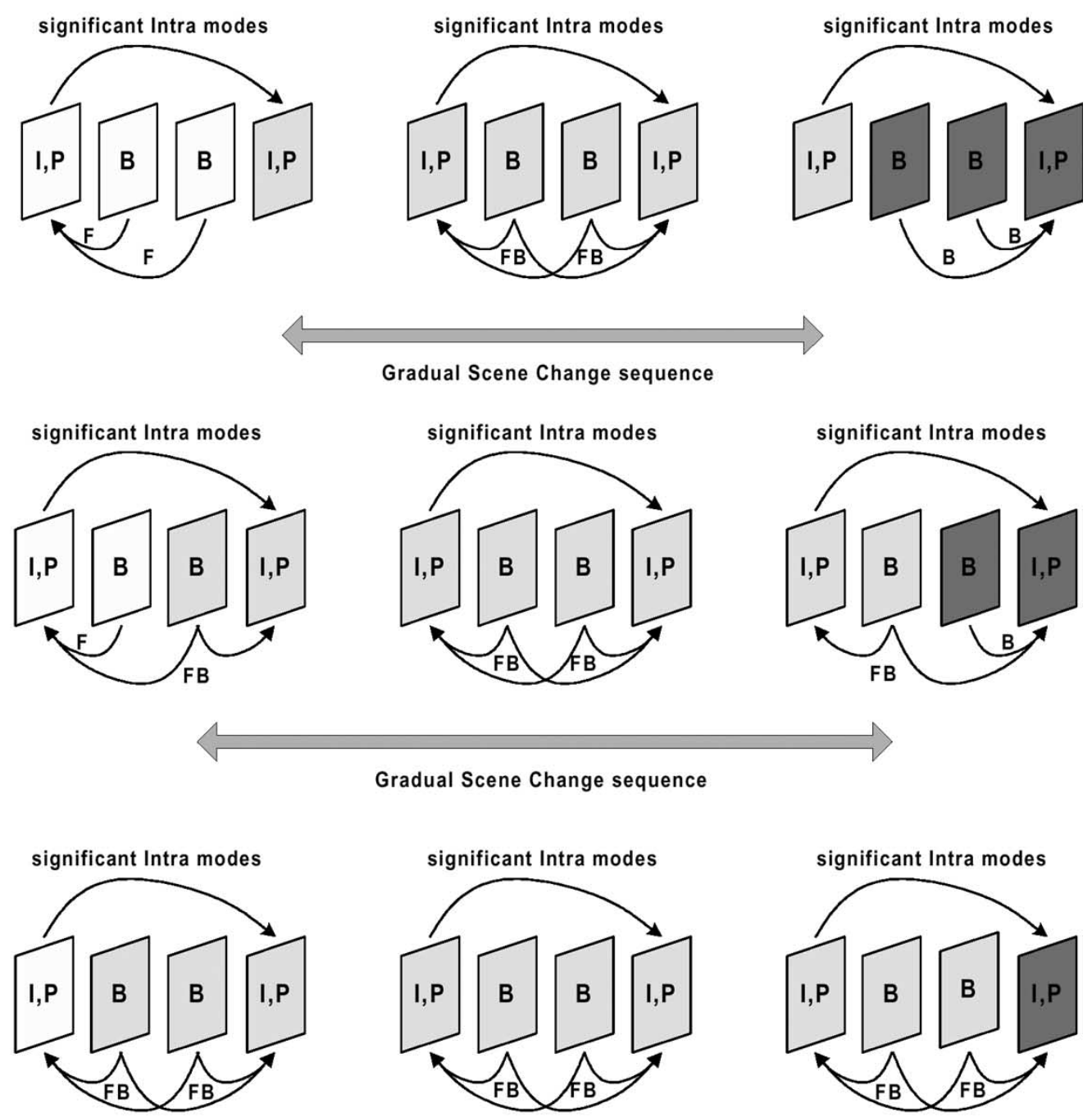

Gradual Scene Change sequence
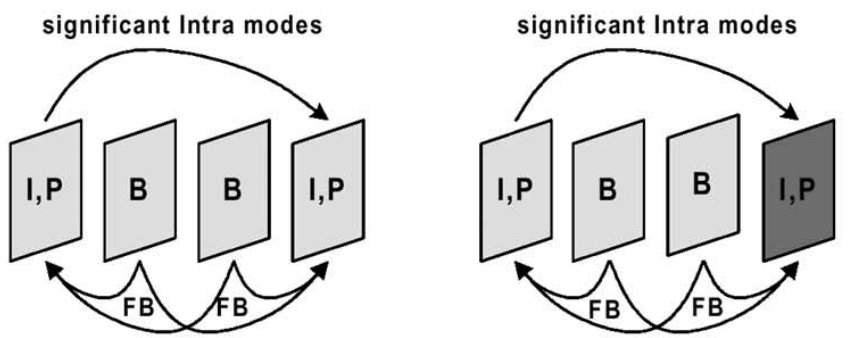

Gradual Scene Change sequence

Fig. 4. Patterns of macroblock type the video sequence involving the gradual scene change.

wait for the second $\mathrm{B}$ frame decoding to complete the scene change detection. In order to overcome this problem, the abrupt scene change detection method is integrated with the method proposed by Meng et al. [28]. The integrated scene change detection is based on the high ratio of forward or backward MB types instead of the concept of SGOPxy [27] when only one B frame is decoded. After the second B frame is decoded, however, the scene change detection is switched back to that based on SGOPxy in order to reduce the probability of false alarm. The high ratio of intracoded MB on the $\mathrm{P}$ frame is still used as an indicator of potential SGOP to speed up the detection. The scene change detection algorithm continuously monitors the $\mathrm{MB}$ type of the $\mathrm{P}$ frame, and high ratio of I mode MB activates the collection mechanism of MB type information of the following B frames. After that, the ratio of forward or backward compensated MBs is compared with a predefined threshold, and immediately the B frame is found as an abrupt scene change frame or not. Furthermore, it is necessary to note that not only our scene change detection method is suitable for this concept of error concealment. Other scene change detections with higher detection accuracy can probably result in better performance of our proposed error concealment algorithm. The reason to adopt the algorithm [27], [28] is because it can be easily embedded in the decoding process and only a very small overhead memory space is needed.

Compared with the abrupt scene change detection, the literatures concerning detection of gradual scene change are fewer. We have also proposed a dissolve sequence detection using the MB information [27]. The overall change of contents between neighboring $\mathrm{P}$ frames and the property of interpolation causes two useful characteristics of MB types. First, a significant number of MBs are intracoded due to the significant change of contents. Second, a dominant number of MBs are interpolative motion compensated owing to the linear property of the dissolve sequences. These two characteristics can be utilized to detect dissolve sequence. In practice, similar to the abrupt scene change, the detection mechanism holds in a waiting state until potential SGOP is found. The comparison between the ratio 


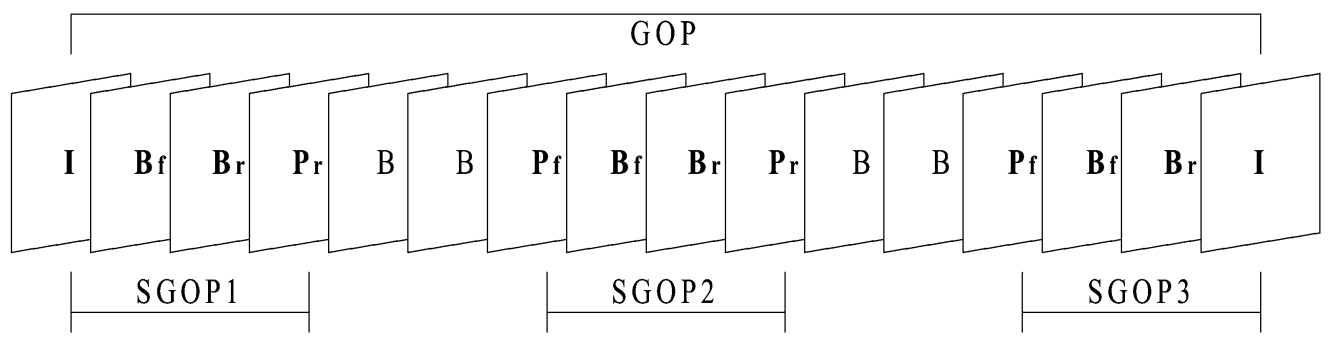

Fig. 5. Frame structure in the MPEG framework.

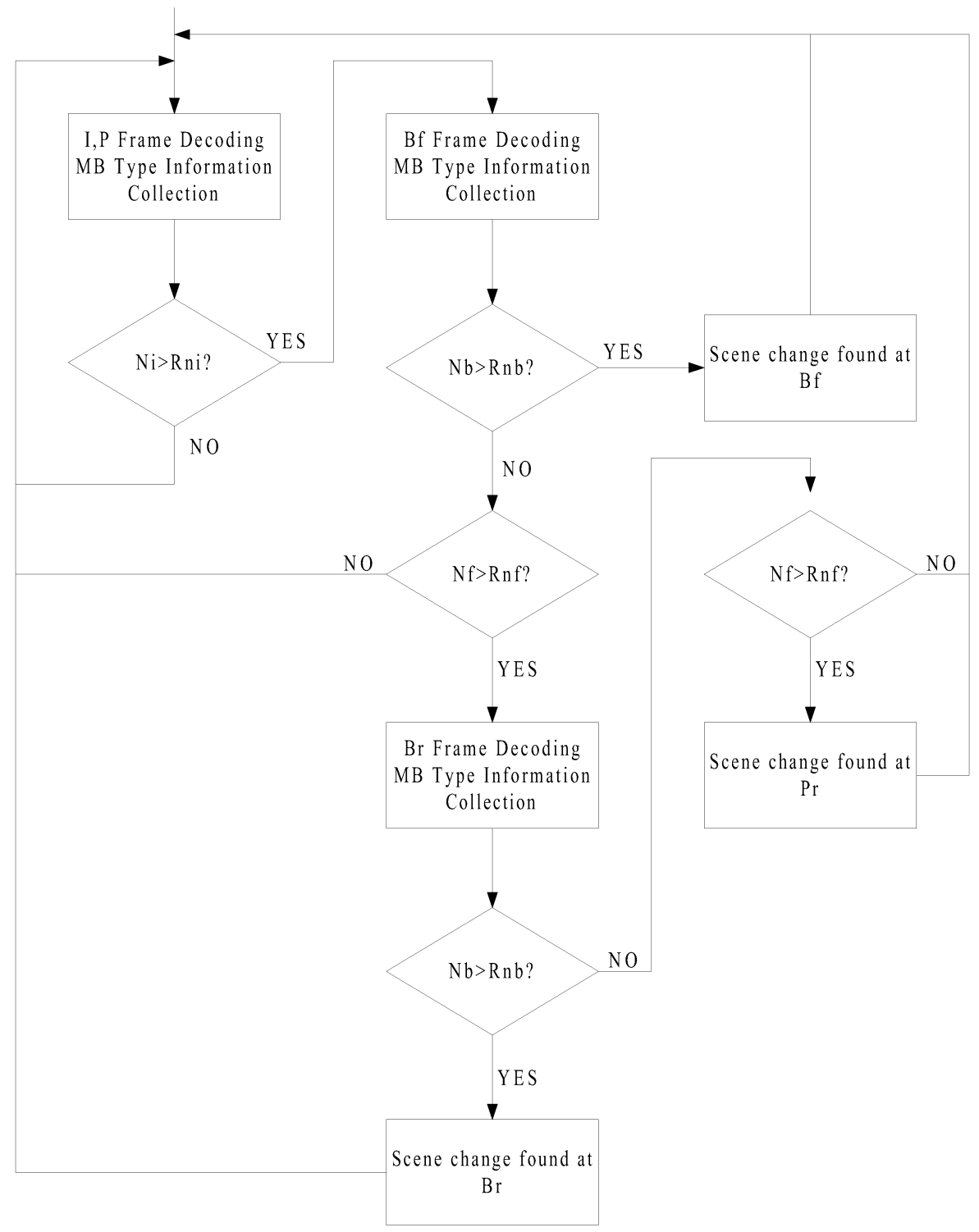

(a)

Fig. 6. (a) Illustration of abrupt scene change detection.

of interpolative compensated MBs and a predefined threshold is performed to detect the gradual scene change. Fig. 6(a) and (b) illustrate the scene change detection algorithms. The scene change detection is activated when the ratio of intracoded MBs of the $\mathrm{P}$ frame is more than a predefined threshold $R_{n i}$. In the activation state, if the ratio of backward prediction MBs is over $R_{n f}$, this $B_{f}$ will be regarded as a scene change frame according to the illustration in Fig. 3. If it is not the case, the scene change detection algorithm checks the next $B_{r}$ frame to see if the second or third pattern in Fig. 3 is fitted. The parameter set of $\left\{R_{n i}, R_{n f}, R_{n b}\right\}$ is set as $\{5 \%, 85 \%, 85 \%\}$ from the study of our past research [27]. As to the gradual scene change, the detection activation mechanism is similar to the abrupt scene change detection. If the ratio of interpolative 


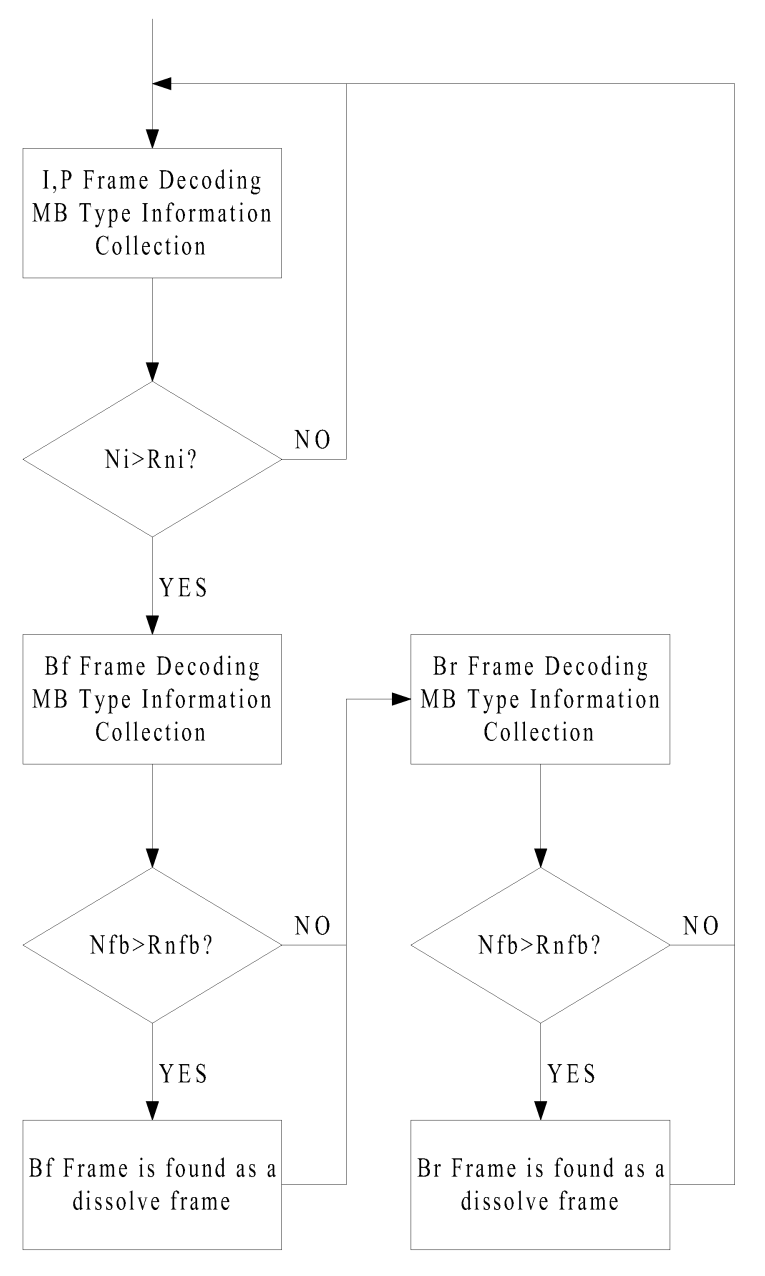

(b)

Fig. 6. (Continued.) (b) Illustration of gradual scene change detection.

MBs of a B frame exceeds a predefined threshold $R_{n f b}$, the B frame will be considered a dissolve frame. $R_{n f b}$ is set as $80 \%$ according to our past proposal.

\section{Proposed Algorithm For ERror CONCEALMENT}

\section{A. Strategy for the Abrupt Scene Change}

When an abrupt scene change occurs, the conventional temporal error concealment algorithm predicts the corrupt MBs to the anchor frame with no temporal correlation at all. It causes the corrupt area entirely in error, and furthermore the error will propagate all the remaining frames of the GOP. Here, we introduce a novel error concealment method to overcome the problem resulting from inadequate temporal error concealment for the abrupt scene change. For simplicity, our method is divided into two parts: error concealment in the $\mathrm{P}$ frame and error concealment in the $\mathrm{B}$ frame. The inequality of the amount of the anchor frames results in different error concealment principles. For P frames, only one anchor P or I frame exists. When an abrupt scene change involved in a SGOP, there is totally no temporal correlation between $P_{r}$ and $P_{f}$ and, once the bit error occurs, the temporal error concealment is no

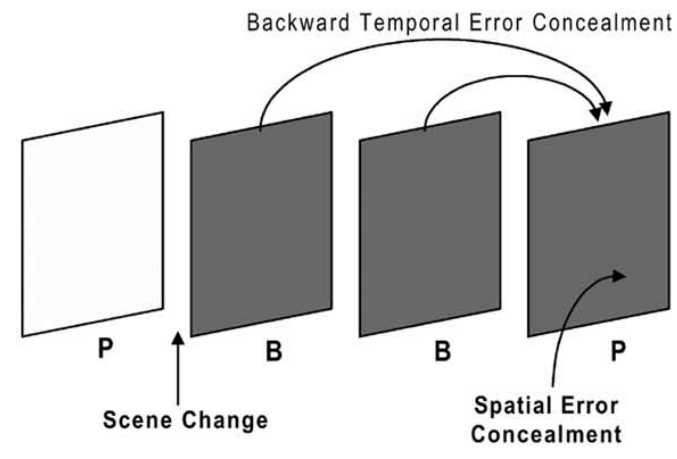

Forward Temporal Error Concealment Backward Temporal Error Concealment
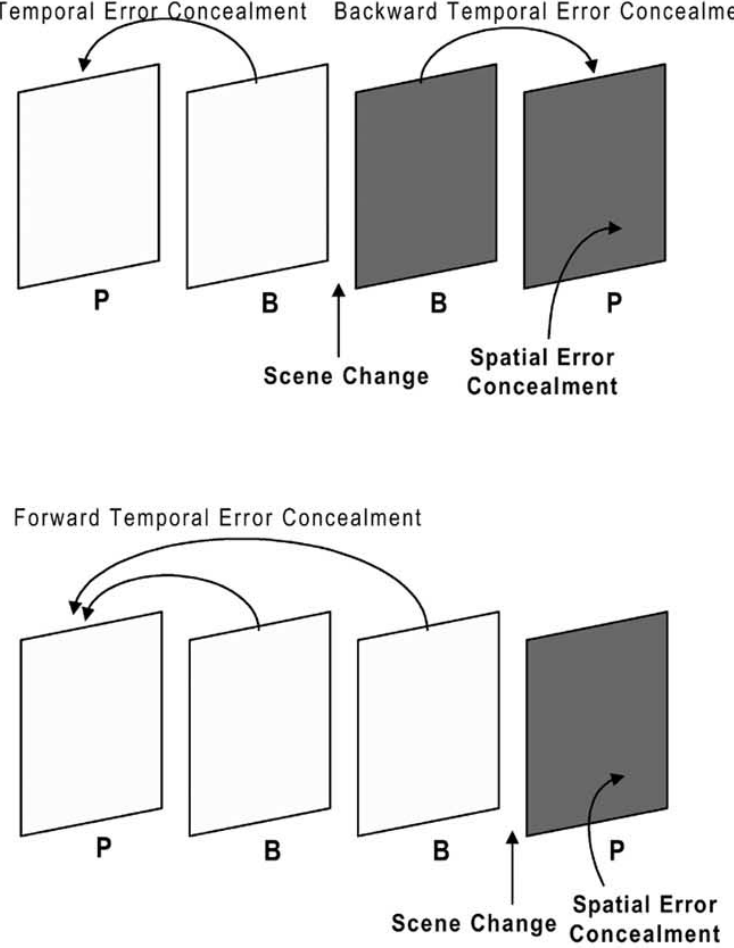

Fig. 7. The illustration of the error concealment method to deal with abrupt scene changes with different scene change locations.

longer adequate. The only remaining choice is spatial error concealment though it is not very satisfactory in performance. Compared with zero temporal correlation between $P_{r}$ and $P_{f}$, the spatial correlation between the corrupt MB and the adjacent MBs is more significant. In our algorithm, once the scene change is detected, the error concealment mechanism of $P_{r}$ is switched from temporal to spatial mode. In our method, the weighted interpolation method [9] is utilized for spatial error concealment (SEC). As to the error concealment of B frames, the prediction direction for concealment of corrupt MBs is decided explicitly according the scene change location. Clearly, the corrupt MB of the B frame in scene change SGOP should temporally concealed by the anchor frame, either an I or $\mathrm{P}$ frame, which belongs to the same shot because the temporal redundancy still exists. If the scene change occurs at $B_{r}$, the corrupt MBs of $B_{f}$ should be temporally concealed by $P_{f}$, and $B_{r}$ by $P_{r}$, respectively. Fig. 7 illustrates the strategy of error concealment of the proposed algorithm. 


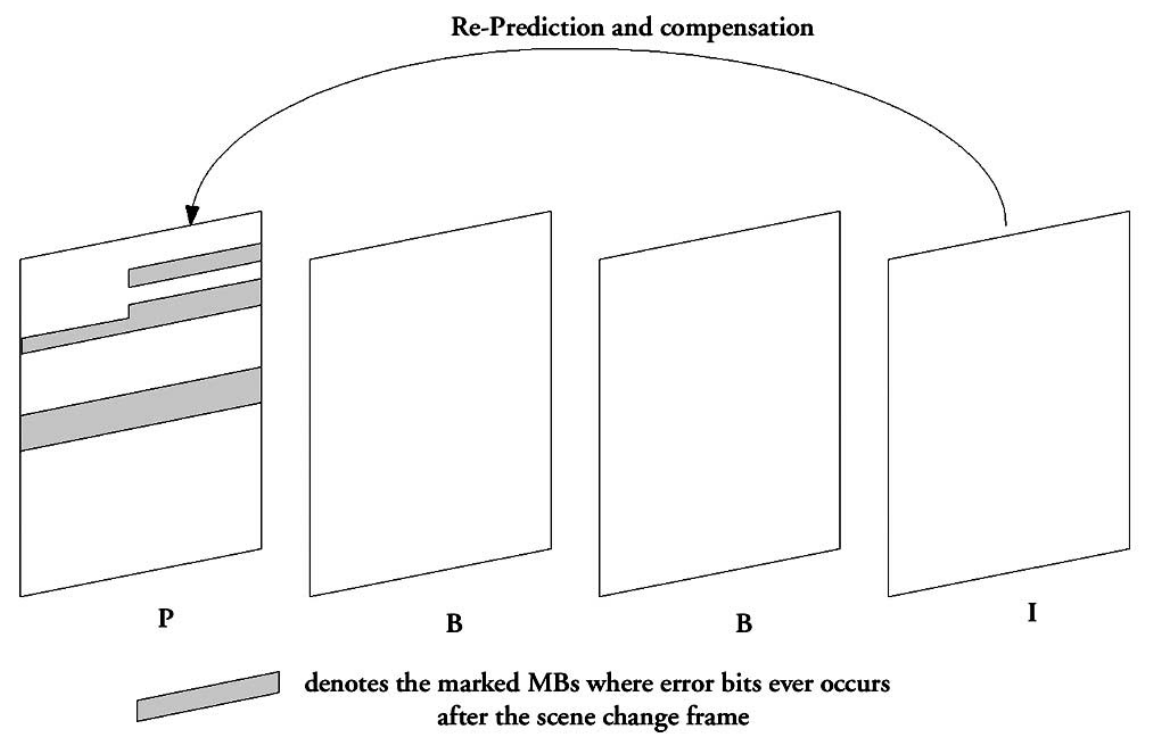

(a)

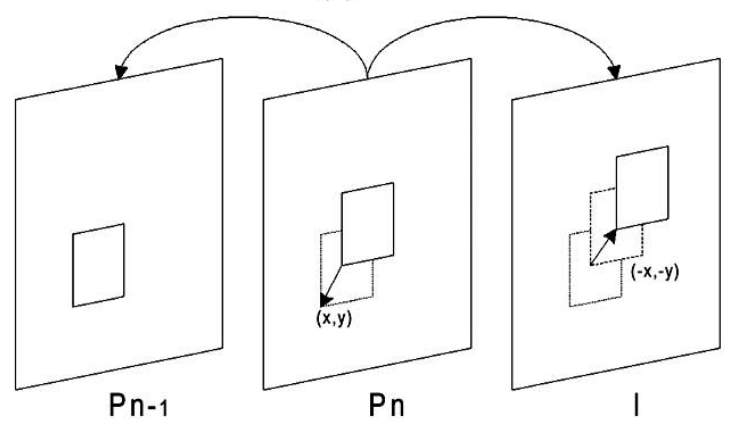

(b)

Fig. 8. (a) Recovering procedure at the last SGOP of the GOP. (b) Illustration of inverse motion vectors for recovering procedure.

In addition, the error propagation due to the scene change stops as the GOP ends. When I frame of the next SGOP is decoded, the impact of scene changes on error concealment is released. This nature introduces a better error concealment mechanism on the last SGOP of the GOP involving an abrupt scene change. The I frame belonging to the next GOP can provide a better reference for concealment. Theoretically, the last $\mathrm{P}$ frame of the GOP, which involves an abrupt scene change, is poor in quality because of the unsatisfactory performance of spatial error concealment and the error propagation. A remedy is proposed by marking the corrupt regions where spatial error concealment is applied, and compensating this region of the last $\mathrm{P}$ frame with the refreshed I frame. This strategy provides a solution to relieve the impact of the scene change on the last SGOP. Fig. 8(a) illustrates this concept and the dark regions denote the marked regions, where the bit errors ever occur at the Pr frame in the scene change SGOP. In other words, MBs of the marked regions have ever been applied the spatial error concealment. As many researches conclude, spatial error concealment is less effective than temporal error concealment. Thus, the I frame of the next GOP provides an opportunity to recover from this ineffectiveness. For the purpose of easy implementation and low computation complexity, the motion vectors of the re-prediction adopt the inverse motion vectors of the marked MBs under the assumption that the motion is linear in the time axis. Fig. 8(b) illustrates this idea. It is noted that the motion vectors of corrupted regions are calculated from the neighboring blocks that are successfully decoded without error. This method is the same as that of MCTEC. Although, by applying reprediction and compensation, the impact of the scene change is blocked outside the last SGOP, the improvement is not free and some efforts must be made. First, extra memory space for storing the locations of marked MBs is needed. Compared with the memory space for reference frames, however, the extra memory is quite small and acceptable. Furthermore, the most significant influence of proposed algorithm lies in the delay of display. Using this algorithm, the last $\mathrm{P}$ frame of the GOP cannot display immediately after decoding the front two B frames. It has to wait until I frame is decoded. This procedure requires extra virtual buffer, whose size is about the bit rate of a $\mathrm{P}$ frame, for real-time data transmission. Fig. 9 shows that the error concealment results based on MCTEC and our proposed method. Compared with MCTEC, the proposed method makes great improvement.

\section{B. Strategy for Gradual Scene Change}

The gradual scene change resulting from dissolve effect takes a sequence of frames to switch from one scene to another. 


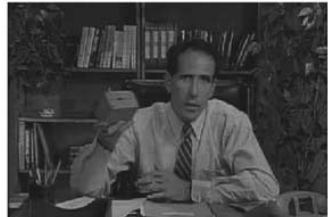

Frame 7

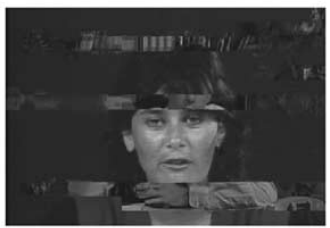

Frame 11

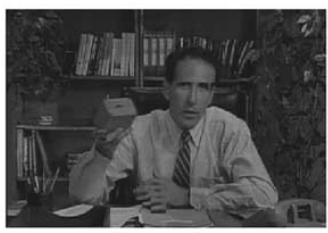

Frame 7

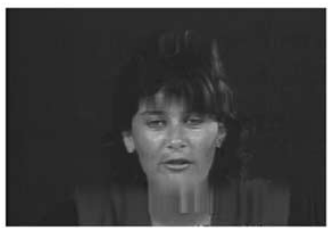

Frame11

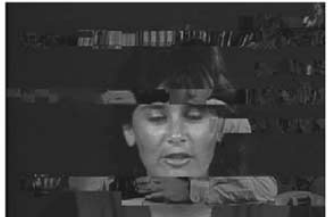

Frame8

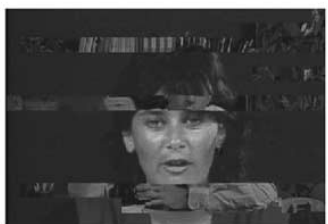

Frame 12

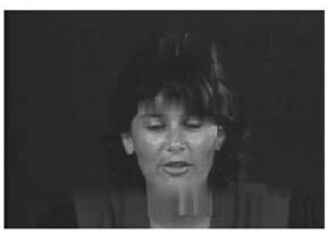

Frame8

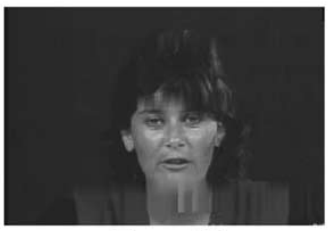

Frame12

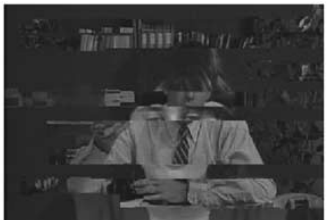

Frame9

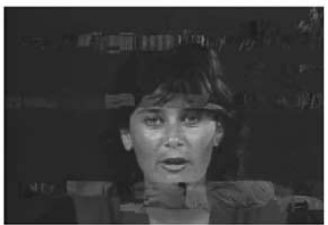

Frame 13

(a)

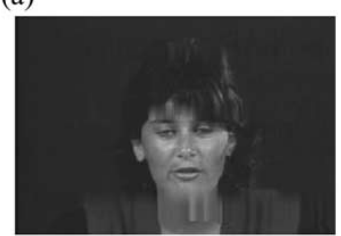

Frame9

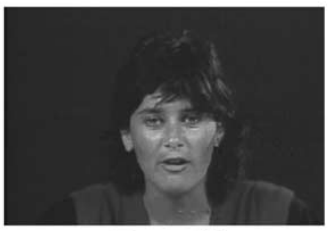

Frame13

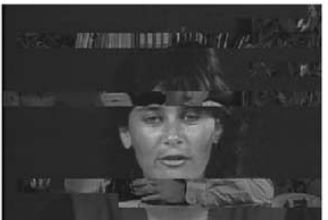

Frame10

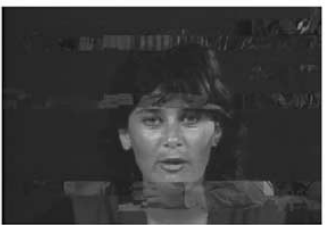

Frame14

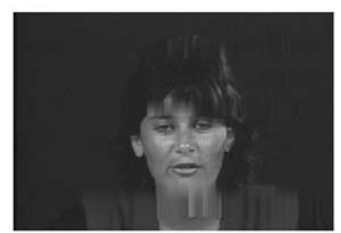

Frame 10

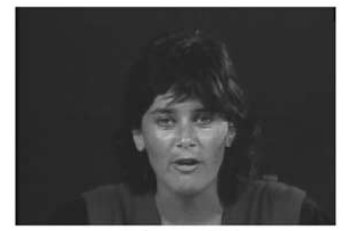

Frame 14

(b)

Fig. 9. (a) Error concealment results using MCTEC. (b) Error concealment results using proposed method (scene change at frame 8).

Within the gradual scene change sequence, the temporal correlation is less significant and the temporal error concealment is not practical when errors occur. To handle this problem, we introduce a novel extrapolation and interpolation error concealment (EIEC) algorithm. The gradual scene change algorithm described in Section III acts as an initial step toward EIEC algorithm. As Fig. 6(a) illustrates, the dissolve sequence is detected once the ratio of interpolative MBs exceeds a predefined threshold in the potential SGOP where $P_{r}$ has significant number of intracoded MBs. In the first SGOP involved in the dissolve sequence, the corrupt MBs of $P_{r}$ are still applied the temporal error concealment. However, some improvement can be made on the error MBs of B frames since there are two possible reference frames. Based on the interpolation property of dissolve sequence, the mathematical relation between $P_{f}$, $B_{f}$ and $P_{r}$ should follow:

$$
B_{f}=x_{B} \cdot P_{f}+\left(1-x_{B}\right) \cdot P_{r}
$$

where $x_{B}$ is the parameter of interpolation and can be calculated by the following (2):

$$
x_{B}=\frac{1}{N} \sum_{B_{f i} \in B_{f c}, P_{r i} \in P_{r c}, P_{f i} \in P_{f c}} \frac{B_{f i}-P_{r i}}{P_{f i}-P_{r i}}
$$

where $P_{f c}, P_{r c}$ and $B_{f c}$ represent the region where no error MB occurs on all three frames and $N$ represents the number of the total pixels of the region. In practice, the parameter $x_{B}$ can be obtained from a small number of selected MBs instead of all MBs. By applying the parameter $x_{B}$ and the property of interpolation, the corrupt MBs can be concealed using (1). By contrast, conventional temporal error concealment is not sufficient for handling the gradual scene change because neither $P_{r}$ nor $P_{f}$ alone is suitable for concealment.

Concerning the error concealment of $P$ frames, a more complex algorithm is proposed. A group of $\mathrm{P}$ frames, denoted as $P_{n-1}, P_{n}$ and $P_{n+1}$, across two adjacent SGOPs involved in the gradual scene change also follows the property of linearity. As a result, similar to B frames, the parameter $x_{p}$ of interpolation can be found from (5), where $P_{(n-1) c}, P_{n c}$, and $P_{(n+1) c}$ represent the region where no error $\mathrm{MB}$ occurs on all three consecutive $P$ frames. See (3)-(5) at the bottom of the next page.

For error MBs of $P_{n+1}$, extrapolation from $P_{n-1}$ and $P_{n}$ by (4) can provide very adequate concealed MBs. On the other hand, however, the interpolation of $P_{n+1}$ and $P_{n+1}$ by (3) to conceal the error MBs of $P_{n}$ is not so effective because the error of $P_{n}$ will propagates to $P_{n+1}$. Fortunately, the significant ratio of intracoded MBs of $P_{n+1}$ provides some refreshed MBs co-located with the error MBs of $P_{n}$. It makes interpolation error concealment at the MB, where intracoding applied in $P_{n+1}$, become valid. The EIEC algorithm is illustrated in Figs. 10 and 11. In the conventional decoding process, the memory space for $P_{n-1}$ can be released immediately after all 

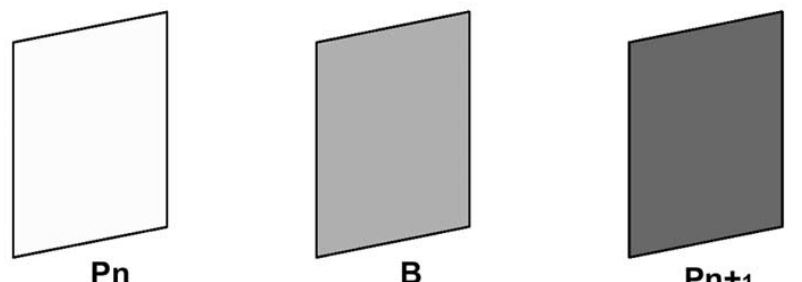

Pn+1
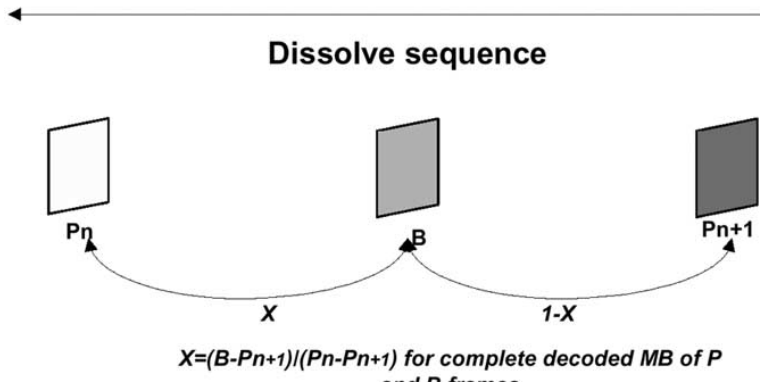

and $B$ frames

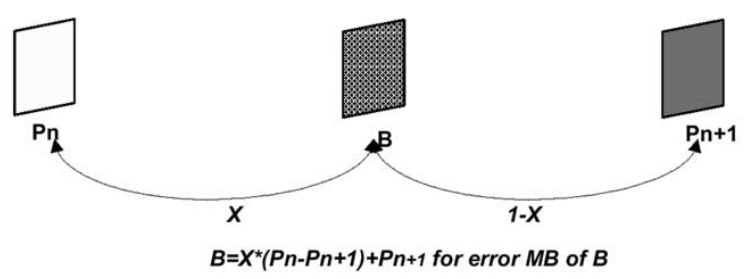

Fig. 10. Interpolated temporal error concealment method to handle B frames in the dissolve sequence.

B frames belonging to the same SGOP are decoded. It seems that some overhead memory space is needed to store $P_{n-1}$ because $P_{n-1}$ has to be reserved for the purpose of EIEC. Actually, the memory space to store two reference frames and current decoding frame, i.e., the memory to store three frames, are used for decoding the B frames. It implies that overhead memory is not needed for the storage of $P_{n-1}, P_{n}$ and current decoding $P_{n+1}$. Our method simply makes memory utilization of the decoding process of the $P$ frame similar to the $B$ frame.

However, our method still has some side effects. $P_{n}$ has to delay its display until EIEC algorithm is done. In other words, $P_{n}$ cannot display until $P_{n+1}$ is decoded. Similar to the abrupt scene change, EIEC also requires larger virtual buffer for real-time application. Fig. 12 shows the experiment results of EIEC and conventional MCTEC. In Fig. 12(a), the upper three images are used to show the effectiveness of EIEC and the lower three are used to show how the last SGOP recovering works.

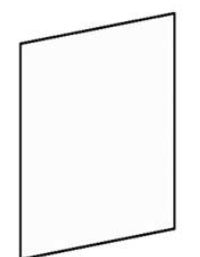

$\mathrm{Pn}-1$

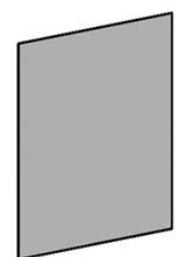

$\mathrm{Pn}$

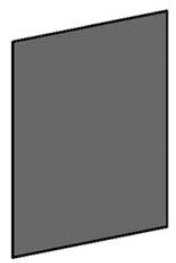

$P n+1$

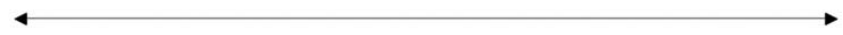

Dissolve sequence

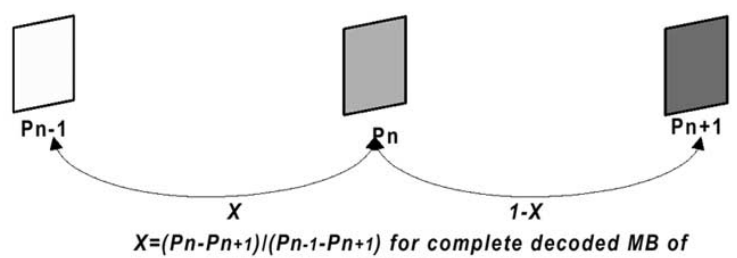
$P$ frames
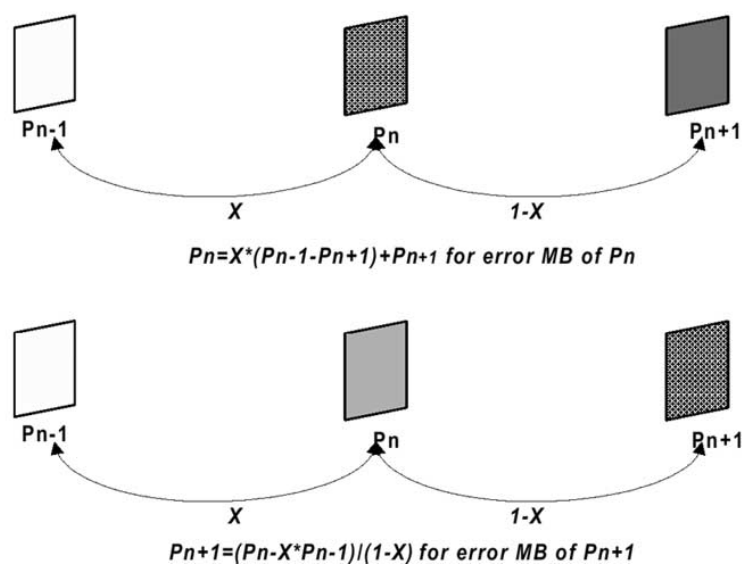

Fig. 11. Illustration of interpolated and extrapolated temporal error concealment to handle $\mathrm{P}$ frames in the dissolve sequence.

\section{Simulation Results}

\section{A. Abrupt Scene Change}

In order to examine the performance of the proposed method, we have edited several video sequences from common-used "Miss America" and "Sales" and designed a series of experiments. The video format is SIF $(352 \times 240)$ and bit rate is $4 \mathrm{Mbps}$. In each experiment, three methods, which include BREC, MCTEC, Weighted Interpolation Spatial Error Concealment (WISEC) [9], and the proposed algorithm, are

$$
\begin{aligned}
P_{n} & =x_{P} \cdot P_{n-1}+\left(1-x_{P}\right) \cdot P_{n+1} \\
P_{n+1} & =\frac{1}{1-x_{P}} \cdot P_{n}+\frac{x_{P}}{1-x_{P}} \cdot P_{n-1} \\
x_{P} & =\frac{1}{N} \sum_{P_{n i} \in P_{n c}, P_{(n+1) i} \in P_{(n+1) c}, P_{(n-1) i} \in P_{(n-1) c}} \frac{P_{n i}-P_{(n+1) i}}{P_{(n+1) i}-P_{(n-1) i}} .
\end{aligned}
$$




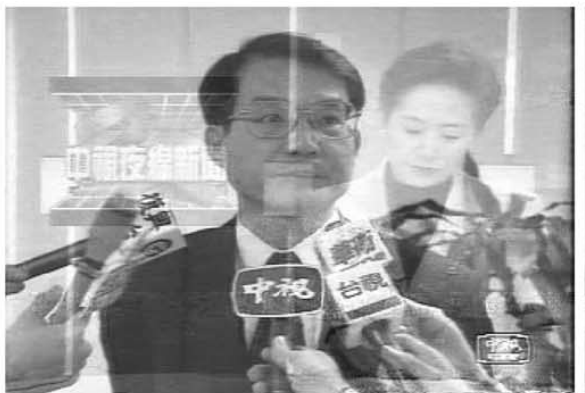

Frame 5

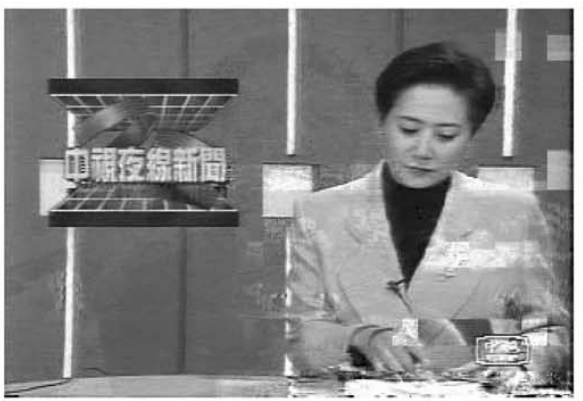

Frame 10

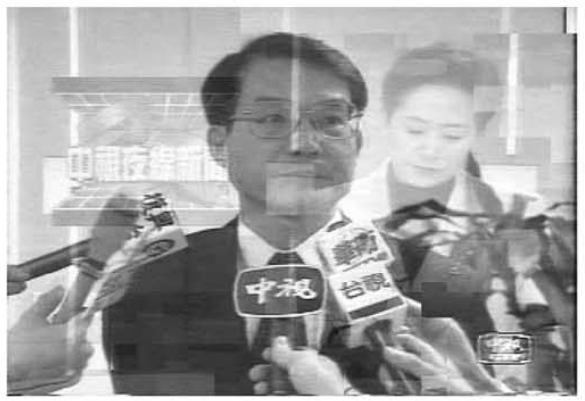

Frame 5

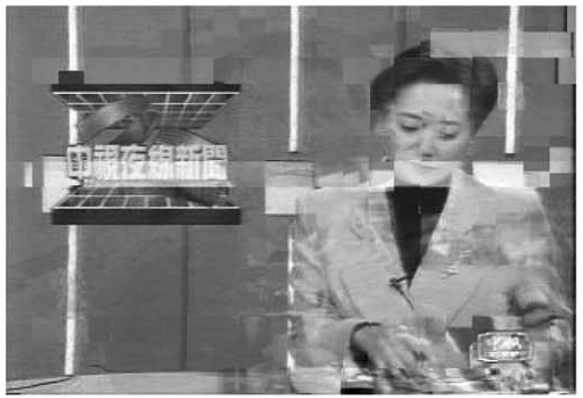

Frame 10

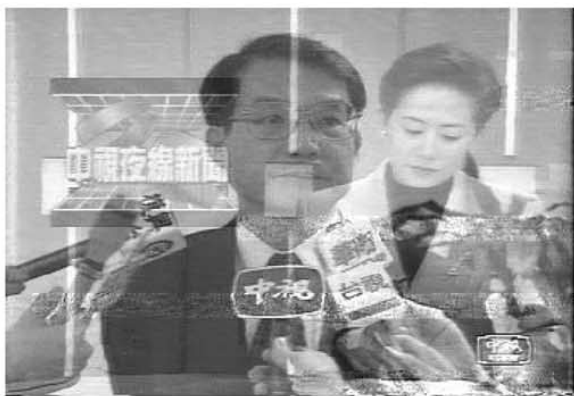

Frame 6

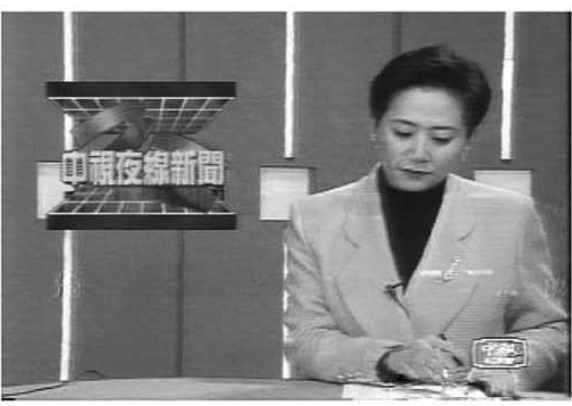

Frame 12

(a)

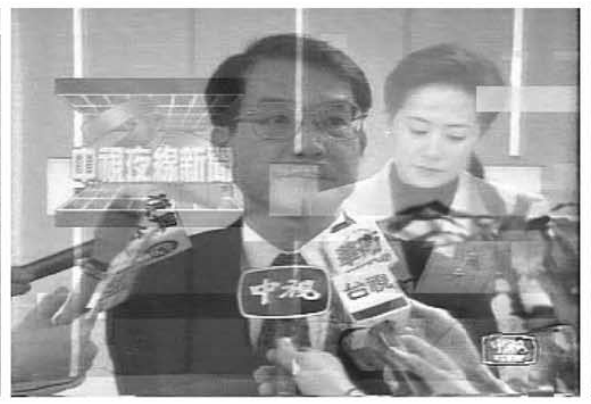

Frame 6

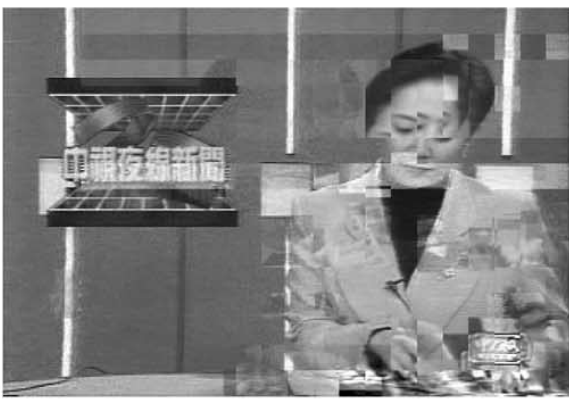

Frame 12

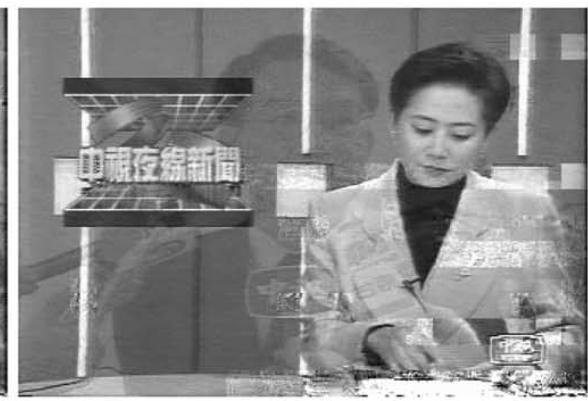

Frame9

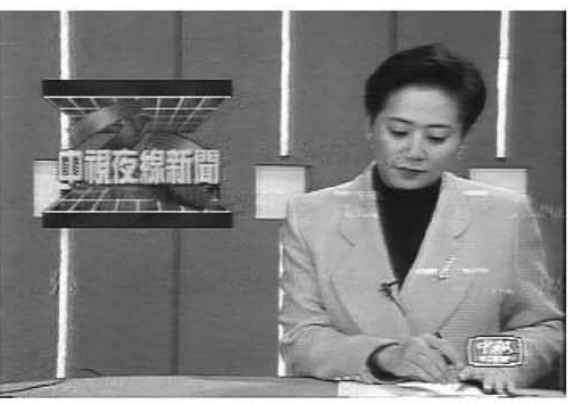

Frame13

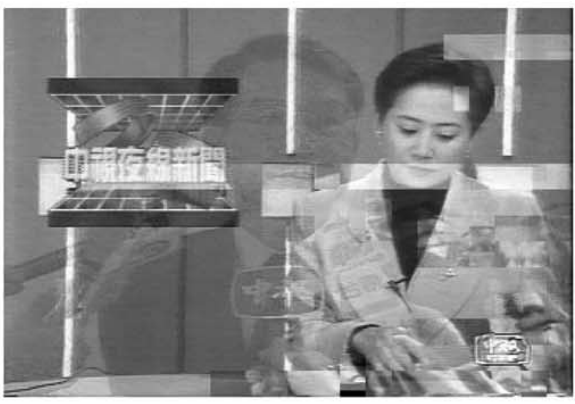

Frame 9

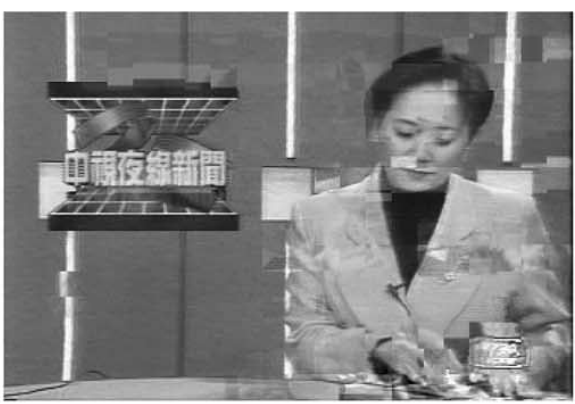

Frame 13

(b)

Fig. 12. (a) Error concealment results using EIEC method. (b) Error concealment results using MCTEC (dissolve from frame 2 to 11).

simulated and compared. In advance of the description of our experiments, it is necessary to define the error model used in the experiments. Error llock (EB) is used to represent that a $\mathrm{MB}$ is corrupted owing to some bit errors that result in invalid codewords and corrupt the MB decoding. Once an MB is corrupted, the following MBs in the same slice will also be lost because no resynchronization point can be found. The decoding process will not recover until the slice header of the next slice is encountered. For example, if the first MB of a specific slice is corrupted, all MBs of the slice will be lost. In this paper, block error rate (BER) is adopted and applied to all experiments. A different ratio, which is defined as ratio of error block (REB), is also introduced to make the clear discrimination from BER. The definitions of BER and REB 
TABLE I

Simulation Results of Different ScENe Change Location and DifFERENT Block ERRoR Rate (in dB)

\begin{tabular}{|c|c|c|c|c|c|c|c|c|c|c|c|c|c|c|c|}
\hline Location & 1 & 2 & 3 & 4 & 5 & 6 & 7 & 8 & 9 & 10 & 11 & 12 & 13 & 14 & Mean \\
\hline $\mathrm{BER}=0.5 \%$ & 39.98 & 39.81 & 39.73 & 40.16 & 39.9 & 39.65 & 39.48 & 39.09 & 38.77 & 38.45 & 38.07 & 37.68 & 37.63 & 37.17 & 38.97 \\
\hline $\mathrm{BR}$ & .61 & 32.46 & 33.27 & 32.7 & 34.00 & 34.08 & $\begin{array}{l}.42 \\
\end{array}$ & 4.11 & 4.41 & 34.41 & 34.61 & 35.43 & 34.69 & 4.88 & 386 \\
\hline MC1 & .76 & 32.67 & 33.59 & 32.72 & 33.97 & 34.1 & 4.25 & 3.84 & 34.21 & 33.99 & 34.34 & 35.09 & 34.39 & 34.3 & 3.73 \\
\hline WISEC & 5.23 & 34.98 & 34.63 & 34.39 & 33.94 & 33.34 & 33.10 & 32.25 & 32.20 & 31.45 & 31.29 & 28.56 & 29.06 & 28.83 & 32.38 \\
\hline Prop & .38 & 37.52 & 37.78 & 37.88 & 37.76 & 37.76 & 37.81 & 37.45 & 37.08 & 36.51 & 36.29 & 36.17 & 35.69 & 34.97 & 37.00 \\
\hline $\mathrm{BER}=$ & .98 & 39.81 & 39.73 & 40.16 & 39.9 & 39.65 & 39.48 & 39.09 & 38.77 & 38.45 & 38.07 & 37.68 & 37.63 & 37.17 & 8.97 \\
\hline BREC & 28.02 & 28.56 & 31.09 & 29.29 & 30.40 & 31.37 & 30.89 & 32.33 & 32.67 & 33.03 & 32.74 & 33.31 & 33.33 & 33.52 & 31.47 \\
\hline MCTEC & 8.26 & 28.67 & 31.12 & 29.32 & 30.36 & 31.21 & 30.69 & 31.93 & 32.14 & 32.58 & 32.23 & 32.68 & 32.48 & 32.83 & 31.18 \\
\hline WIS & .23 & 31.79 & 32.48 & 31.65 & 31.23 & 30.32 & 30.22 & 28.96 & 28.69 & 26.97 & 28.06 & 29.29 & 25.68 & 25.52 & 29.58 \\
\hline Prop & 35.4 & 36.16 & 36.58 & 36.27 & 36.26 & 36.54 & 36.25 & 36.05 & 35.94 & 35.51 & 34.64 & 34.64 & 34.07 & 33.72 & $\mathbf{3 5 . 5 7}$ \\
\hline $\mathrm{BER}=2.0 \%$ & 39.98 & 39.81 & 39.73 & 40.16 & 39.9 & 39.65 & 39.48 & 39.09 & 38.77 & 38.45 & 38.07 & 37.68 & 37.63 & 37.17 & 38.97 \\
\hline BREC & 3.47 & 24.41 & 26.12 & 26.47 & 27.97 & 28.77 & 28.8 & 29.81 & 30.08 & 30.31 & 31.11 & 31.19 & 31.12 & 31.53 & 28.65 \\
\hline $\mathrm{MC}$ & 23.76 & 24.6 & 26.36 & 26.52 & 27.78 & 28.63 & 28.21 & 29.25 & 29.52 & 29.29 & 30.29 & 30.42 & 30.08 & 30.58 & 28.24 \\
\hline WISEC & 8.54 & 28.19 & 28.72 & 27.40 & 26.90 & 26.39 & 26.42 & 24.65 & 25.32 & 25.11 & 23.51 & 23.59 & 23.06 & 22.34 & 25.73 \\
\hline Proposed & 32.39 & 32.73 & 33.55 & 33.73 & 34.05 & 34.55 & 34.05 & 34.13 & 33.86 & 33.12 & 33.13 & 32.82 & 32.29 & 31.64 & 33.29 \\
\hline $\mathrm{BER}=$ & .98 & 39.81 & 39.73 & 40.16 & 39.9 & 39.65 & 39.48 & 39.09 & 38.77 & 38.45 & 38.07 & 37.68 & 37.63 & 37.17 & 38.97 \\
\hline BREC & 1.70 & 23.09 & 24.48 & 24.58 & 26.21 & 27.39 & 27.75 & 28.05 & 28.55 & 28.91 & 29.33 & 29.25 & 29.19 & 29.98 & 27.03 \\
\hline MCTEC & 2.00 & 23.14 & 24.54 & 24.77 & 26.57 & 27.03 & 26.88 & 27.27 & 26.40 & 27.85 & 27.92 & 28.34 & 27.98 & 28.89 & 26.40 \\
\hline & .61 & 26.09 & 26.13 & 24.35 & 24.17 & 24.54 & 24.19 & 24.00 & 23.17 & 22.88 & 21.85 & 21.43 & 21.20 & 20.19 & 23.63 \\
\hline Proposed & 30.67 & 31.85 & 31.25 & 31.49 & 32.21 & 32.57 & 32.59 & 32.24 & 30.79 & 31.71 & 30.66 & 30.89 & 30.27 & 29.97 & 31.37 \\
\hline
\end{tabular}

TABLE II

Simulation Results of DifFERENT Contents of AbRuPT ScENe Change (in dB)

\begin{tabular}{|c|c|c|c|c|c|c|c|c|c|c|c|c|c|c|c|c|c|}
\hline BER & $0 \%$ & \multicolumn{4}{|c|}{$0.5 \%$} & \multirow{2}{*}{\multicolumn{4}{|c|}{$\frac{1.0 \%}{10.8 \%}$}} & \multicolumn{4}{|c|}{$2.0 \%$} & \multicolumn{4}{|c|}{$3.0 \%$} \\
\hline REB & $0 \%$ & & 5.5 & $3 \%$ & & & & & & & 20. & $1 \%$ & & & 28. & $1 \%$ & \\
\hline Method & M0 & M1 & M2 & M3 & M4 & M1 & $\mathrm{M} 2$ & M3 & M4 & M1 & M2 & M3 & M4 & M1 & M2 & M3 & M4 \\
\hline Miss $>>$ Sales & 37.78 & 34.21 & 34.32 & 32.45 & 35.77 & 32.63 & 32.84 & 27.15 & 34.37 & 29.65 & 29.77 & 25.66 & 31.99 & 28.17 & 28.08 & 24.53 & 29.86 \\
\hline Miss $>>$ Pingpong & 36.84 & 31.61 & 31.89 & 30.06 & 34.72 & 29.40 & 32.98 & 25.78 & 33.27 & 26.95 & 27.31 & 23.21 & 30.96 & 24.50 & 24.66 & 22.87 & 28.72 \\
\hline Miss $>>$ Windmill & 33.87 & 29.64 & 30.03 & 28.06 & 32.02 & 27.53 & 28.15 & 23.83 & 30.86 & 24.71 & 25.20 & 20.22 & 28.80 & 21.91 & 22.21 & 19.72 & 26.77 \\
\hline Sales $>>$ Miss & 39.09 & 34.11 & 33.84 & 33.10 & 37.45 & 32.33 & 31.93 & 28.96 & 36.05 & 29.81 & 29.25 & 24.65 & 34.13 & 28.05 & 27.27 & 24.00 & 32.24 \\
\hline Sales $>>$ Pingpong & 35.51 & 30.93 & 30.96 & 28.42 & 33.17 & 28.86 & 28.82 & 26.68 & 31.91 & 26.57 & 26.19 & 22.45 & 29.42 & 24.69 & 24.98 & 21.76 & 26.75 \\
\hline Sales $>>$ Windmill & 32.48 & 28.64 & 28.84 & 27.11 & 30.59 & 26.28 & 26.32 & 24.87 & 28.99 & 24.41 & 24.56 & 21.32 & 27.12 & 21.01 & 21.31 & 20.45 & 24.33 \\
\hline Pingpong >>Miss & 38.28 & 30.46 & 30.82 & 30.07 & 36.15 & 26.73 & 27.11 & 25.29 & 35.03 & 23.75 & 24.20 & 20.59 & 32.02 & 21.23 & 21.77 & 19.76 & 30.18 \\
\hline Pingpong $>>$ Sales & 35.67 & 29.56 & 29.75 & 27.95 & 33.04 & 27.64 & 27.95 & 24.96 & 31.61 & 23.77 & 24.23 & 20.05 & 28.62 & 20.14 & 20.24 & 19.65 & 25.02 \\
\hline P'pong $>>W^{\prime}$ 'mill & 31.28 & 27.32 & 27.90 & 26.39 & 28.91 & 25.97 & 26.79 & 22.90 & 28.01 & 23.01 & 23.73 & 19.89 & 25.32 & 19.84 & 20.54 & 19.40 & 22.99 \\
\hline Windmill $>>$ Miss & 36.87 & 26.31 & 27.61 & 25.23 & 34.67 & 22.89 & 24.58 & 21.54 & 33.62 & 19.16 & 21.11 & 18.21 & 31.03 & 18.46 & 18.42 & 18.01 & 27.43 \\
\hline Windmill $>>$ Sales & 33.64 & 25.18 & 26.45 & 24.76 & 31.05 & 22.87 & 24.48 & 21.16 & 29.75 & 19.06 & 21.06 & 18.62 & 27.02 & 18.44 & 18.66 & 18.35 & 24.20 \\
\hline W'mill>>P'pong & 32.71 & 26.64 & 2819 & 25.17 & 30.56 & 23.75 & 25.78 & 21.78 & 28.78 & 20.49 & 22.64 & 18.90 & 26.11 & 18.97 & 19.23 & 18.44 & 24.44 \\
\hline
\end{tabular}

are shown in the (6) and (7). According to our experiments, $0.5 \%$ BER could result in about 5\% REB:

$$
\begin{aligned}
& \mathrm{BER}=\operatorname{Prob}(M B=E B) \\
& \mathrm{REB}=\frac{\text { Number of } E B}{\text { Number of } M B} .
\end{aligned}
$$

In our experiments, for each sequence and some selected BER, 100 simulations are made and the average PSNR is obtained. First, we tried to find out the influence of the location of the scene change in a GOP. Thirteen video sequences with different locations of scene change which switched the scene from "sales" to "miss" are edited and three methods based on different bit error rates are applied. From Table I, it is obvious that the earlier the scene change occurs in a GOP, the greater improvement the proposed method makes. If the scene change occurs at the first B frame in the SGOP, the difference between conventional and proposed method can even be as large as 6.5 to $8.5 \mathrm{~dB}$. It is out of question because the early error propagates more frames in a GOP. Second, a set of the combination of different contents at the two sides of the scene change is experimented with the fixed scene change point at frame 8. Simulation results in Table II show that the sequence of the scene change to "Miss America" benefits the greatest improvement about 5 to $10 \mathrm{~dB}$ PSNR from the proposed algorithm. It is not difficult to understand that homogenous background of "Miss America" sequence make the spatial error concealment more effective. Simulation results of several clips of video sequences, which are digitized from news reports from China Television (CTV) channel in Taiwan with 127 scene changes, are shown in Table III and Fig. 13 shows performance comparison. It is found that about 3 to $5 \mathrm{~dB}$ in PSNR is gained corresponding to different BER. In all the experiments, it is worthwhile to emphasize that only the GOP with a scene change is taken into consideration and PSNR calculation. It is because the error resulting from poorly concealed block due to the scene change will not propagate to next GOP. 
TABLE III

Simulation Results of Real Video Containing Total 127 Scene Changes

\begin{tabular}{c|c|c|c|c|c}
\hline 127 scene change & No Error $(\mathrm{dB})$ & BREC $(\mathrm{dB})$ & MCTEC $(\mathrm{dB})$ & WISEC $(\mathrm{dB})$ & Proposed $(\mathrm{dB})$ \\
\hline $0.5 \%$ & 36.56 & 31.45 & 31.84 & 29.85 & 34.85 \\
\hline $1 \%$ & 36.56 & 28.85 & 29.35 & 25.24 & 33.01 \\
\hline $2 \%$ & 36.56 & 25.25 & 26.34 & 22.16 & 30.72 \\
\hline $3 \%$ & 36.56 & 23.21 & 24.32 & 20.67 & 27.55 \\
\hline
\end{tabular}

Comparison of Three Error Concealment Method (Abrupt Scene Change)

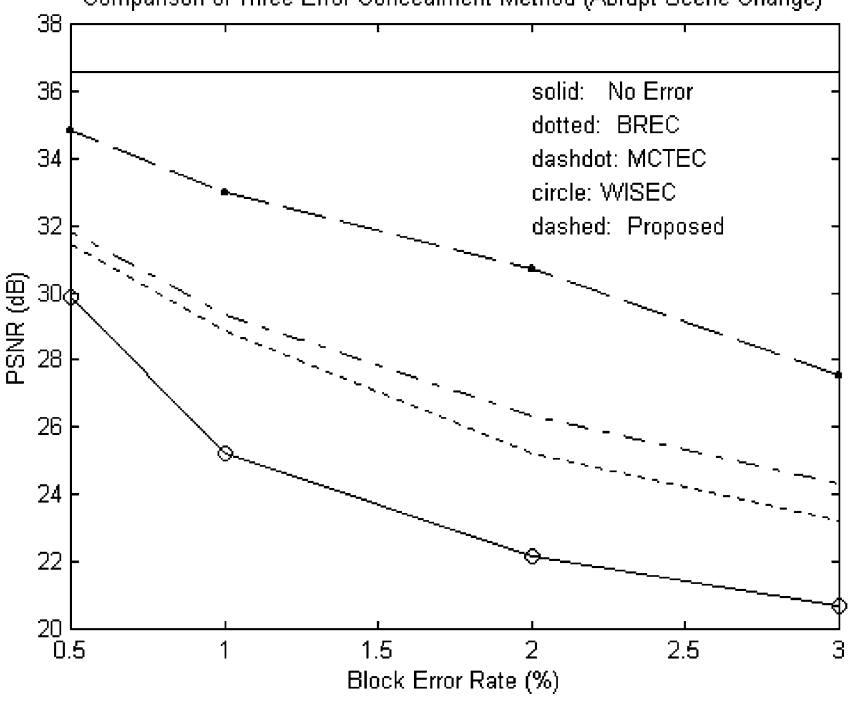

Fig. 13. Plot of comparison of three methods (Abrupt scene change).

\section{B. Dissolve Sequence}

The experiments are simulated based on EIEC and dissolve sequences, different location, length of dissolve sequences and different BER are simulated. As shown in Table IV, similar to the abrupt scene change, the earlier gradual scene change occurs in a GOP, the greater improvement is obtained by EIEC. It is found that 3 to $3.5 \mathrm{~dB}$ PSNR is gained compared with the conventional method. Table V shows the influence of length of the dissolve sequence and 2.5 to $4 \mathrm{~dB}$ gain corresponding to different length. From Table VI, it is also found that EIEC makes significant improvement about $3 \mathrm{~dB}$ PSNR. It is also interesting that MCTEC is more ineffective than BREC. It is because that the dissolve effect makes the motion estimation confused and that chaotic motion vectors make MCTEC very ineffective. Last, 25 dissolve sequences also digitized from CTV news, 5 to 25 in length and 1 to 2 GOPs involved, are extracted from news, and simulation results are shown in Table VII and Fig. 14. Significant improvement about $3 \mathrm{~dB}$ is made by EIEC. It is worthwhile to note again that all simulations are only done on the GOPs, where dissolve effects are involved.

\section{DISCUSSIONS}

\section{A. Overhead Computation Load}

In our error concealment algorithm on the video sequence without scene changes or any special effects, the overhead computation is the threshold comparison only. Compared with the decoding process containing motion compensation and inverse DCT, the overhead computation can almost be neglected. A simple simulation based on a $20 \mathrm{sec}$ news sequence without scene changes shows less than $1 \%$ difference in computation time. As the abrupt scene change occurs, the overhead computation load includes the weighted interpolation spatial error concealment and the recover backward concealment at the last SGOP. This overhead computation load is acceptably low in contrast with the decoding process. As to EIEC algorithm, the overhead computation comprises the parameter $x_{B}$ and $x_{P}$ calculation, the interpolation and extrapolation computation. It is worthwhile to note the complexity of parameter $x_{B}$ and $x_{P}$ calculation depends on $N$, that is, the number of pixels that are successfully reconstructed. In this paper, we select the first 20 successfully decoded MBs at the same locations in $P_{f}, P_{r}$ and $B_{f}$ and $N$ is $20 \times 16 \times 16$ in our experiments. The simulations of computation load are shown in Table VIII based on a sequence containing 45 frames with one scene change and one dissolve sequence. It is interesting that sometimes computation of the error concealment decoding method is lower than that of error free decoding procedure. It is because that sometimes the motion compensation and DCT inversion is more complex than error concealment mechanism. From Table VIII, we can conclude that the overhead computation load is acceptably low.

\section{B. Overhead Memory}

In the conventional decoding process of MPEG, the memory space to store three frames is required for the forward_reference_frame, backward_reference_frame and current_decoding_frame. In addition to those memory, the overhead memory in our algorithm is used for only marking the macroblocks where errors are found. Compared with the memory space to store reference and current decoding frame, the overhead memory space is insignificant and acceptable.

\section{Impact of False Alarm}

No scene change detection algorithm can guarantee no false alarm to occur. Therefore, the impact of the false alarm of scene change detection should be taken into consideration to evaluate the error concealment method. In order to study the robustness of the proposed algorithm for the false alarm, an extreme test is done and the results are shown in Table IX. A randomly selected frame index is directly regarded as the scene change location in the test video sequence. In other words, the video, which contains no scene change, keeps intact, but one selected frame of it is assumed as a scene change frame to simulate the false alarm. The proposed algorithm is performed on the SGOP containing the false alarm. From Table IX, it is found that very insignificant difference between proposed algorithm and conventional temporal error concealment. Moreover, for practical application, the proposed error conceal algorithm executes only for high ratio of intracoded $\mathrm{MBs}$ of $P$ frame. In this instance, temporal error concealment algorithm is actually more inadequate 
TABLE IV

Simulation Results of Different Starting Locations of Dissolve Sequence (BER $=2 \%$ )

\begin{tabular}{c|c|c|c|c|c}
\hline Location & No Error $(\mathrm{dB})$ & BREC $(\mathrm{dB})$ & MCTEC $(\mathrm{dB})$ & WISEC $(\mathrm{dB})$ & Proposed $(\mathrm{dB})$ \\
\hline $4-8$ & 34.92 & 24.71 & 24.33 & 21.83 & 27.81 \\
\hline $5-9$ & 34.95 & 25.99 & 25.55 & 22.14 & 28.57 \\
\hline $6-10$ & 35.03 & 26.64 & 26.3 & 22.95 & 29.41 \\
\hline $7-11$ & 34.93 & 27.35 & 27.15 & 23.18 & 29.97 \\
\hline $8-12$ & 34.98 & 28.47 & 27.97 & 23.34 & 30.15 \\
\hline
\end{tabular}

TABLE V

Simulation of DifFERENT Length OF DisSOlVE SEQUENCE $(\mathrm{BER}=2 \%)$

\begin{tabular}{c|c|c|c|c|c}
\hline Location & No Error $(\mathrm{dB})$ & BREC $(\mathrm{dB})$ & MCTEC $(\mathrm{dB})$ & WISEC $(\mathrm{dB})$ & Proposed $(\mathrm{dB})$ \\
\hline Seq $\mathrm{A}(6-10)$ & 35.03 & 26.64 & 26.30 & 23.12 & 29.41 \\
\hline Seq $\mathrm{B}(3-13)$ & 36.27 & 25.93 & 24.97 & 21.65 & 29.17 \\
\hline Seq C $(10-20)$ & 36.48 & 25.82 & 25.31 & 21.76 & 29.66 \\
\hline
\end{tabular}

TABLE VI

Simulation Results of DifFERENT Block ERror Rates (DisSOlVe From Frame 6 to 10)

\begin{tabular}{c|c|c|c|c|c}
\hline BER & No Error $(\mathrm{dB})$ & BREC $(\mathrm{dB})$ & MCTEC $(\mathrm{dB})$ & WISEC $(\mathrm{dB})$ & Proposed $(\mathrm{dB})$ \\
\hline $0.5 \%$ & 35.03 & 31.81 & 31.40 & 29.55 & 33.07 \\
\hline $1 \%$ & 35.03 & 29.81 & 29.66 & 26.56 & 32.12 \\
\hline $2 \%$ & 35.03 & 26.64 & 26.30 & 22.19 & 29.41 \\
\hline $3 \%$ & 35.03 & 24.99 & 24.63 & 20.33 & 27.96 \\
\hline
\end{tabular}

TABLE VII

Simulation of Real Sequence Containing 25 Dissolve Sequences (25 Dissolve Sequence Across 32 GOPs)

\begin{tabular}{c|c|c|c|c|c}
\hline BER & No Error $(\mathrm{dB})$ & BREC $(\mathrm{dB})$ & MCTEC $(\mathrm{dB})$ & WISEC $(\mathrm{dB})$ & Proposed $(\mathrm{dB})$ \\
\hline $0.5 \%$ & 34.32 & 30.72 & 30.31 & 27.84 & 33.34 \\
\hline $1 \%$ & 34.32 & 29.61 & 29.44 & 25.71 & 32.27 \\
\hline $2 \%$ & 34.32 & 26.51 & 26.35 & 22.97 & 29.52 \\
\hline $3 \%$ & 34.32 & 23.95 & 23.76 & 20.15 & 27.18 \\
\hline
\end{tabular}

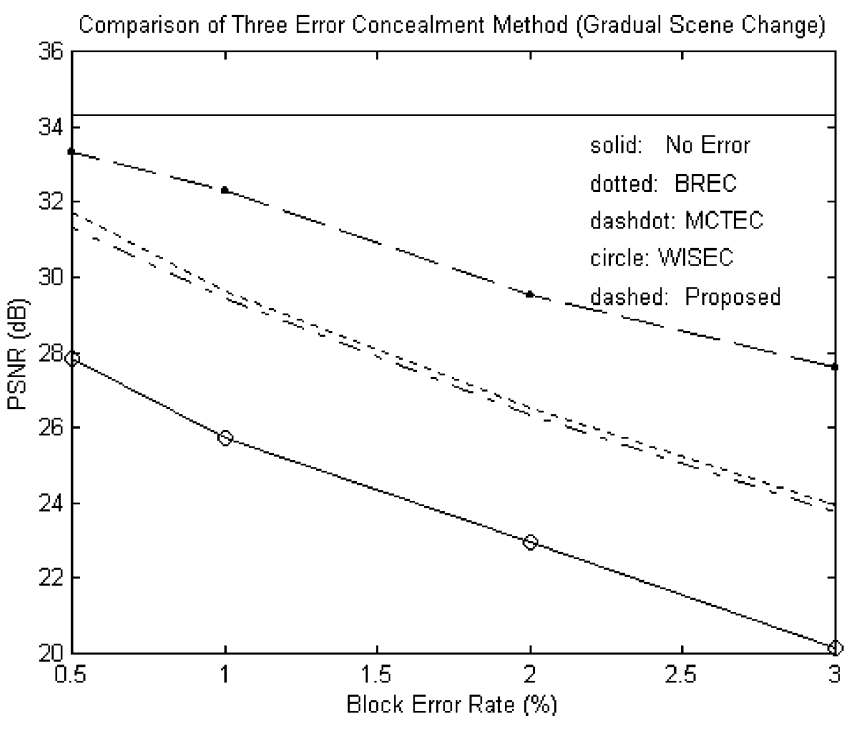

Fig. 14. Plot of comparison of three methods (gradual scene change).

than spatial error concealment. Because of the low percentage of false alarms and acceptable difference shown in Table IX. From Table IX, we can conclude that the impact of false alarm is acceptable. It is also worthwhile to note that, for gradual scene change case, the impact of false alarm is comparatively slight because the parameters of interpolation and extrapolation are calculated based on the contents of the video sequence. Therefore, the concealment result is very close to the original contents when the sequence is simply an ordinary sequence without scene effects.

\section{Other Editing Effects}

In addition to dissolve sequences, there exists another common type of gradual scene change called wipe transition. The wipe transition is much more difficult to detect because its shape, direction and speed can be arbitrary. In this paper, a total solution toward the error concealment to deal with wipe effect is out of our scope, however, we hope to provide an opening attempt. It is worthwhile to note that although our method is not effective in wipe effect but the performance of our algorithm is simply the same as other method when wipe effect is encountered. For the most common pattern of the wipe effect, in which the next scene gradually shows up on the next scene with a specific direction and shape, it can be regarded as a series of frame with a specific area of scene change MBs. This idea, which is based on our past approach [29], is illustrated in Fig. 15. As a result, we can apply our strategy for abrupt scene change to wipe transitions. When the abrupt scene change region cover over a specific ratio of a frame, the error concealment strategy is activated and applied to this detected scene change region. Fig. 16 shows the comparison between the temporal error concealment and our method. It is found the 
TABLE VIII

Simulation Results of COMPUTATION Time (SEC)

\begin{tabular}{c|c|c|c|c|c}
\hline BER & No Error & BREC & MCTEC & WISEC & Proposed \\
\hline $0.5 \%$ & 5.48 & 5.32 & 5.47 & 5.78 & 5.64 \\
\hline $1 \%$ & 5.55 & 5.29 & 5.46 & 5.76 & 5.58 \\
\hline $2 \%$ & 5.41 & 5.28 & 5.46 & 5.81 & 5.6 \\
\hline $3 \%$ & 5.48 & 5.24 & 5.42 & 5.81 & 5.59 \\
\hline $5 \%$ & 5.38 & 5.20 & 5.33 & 5.78 & 5.56 \\
\hline
\end{tabular}

CPU: Pentium 333, 138 Mb RAM

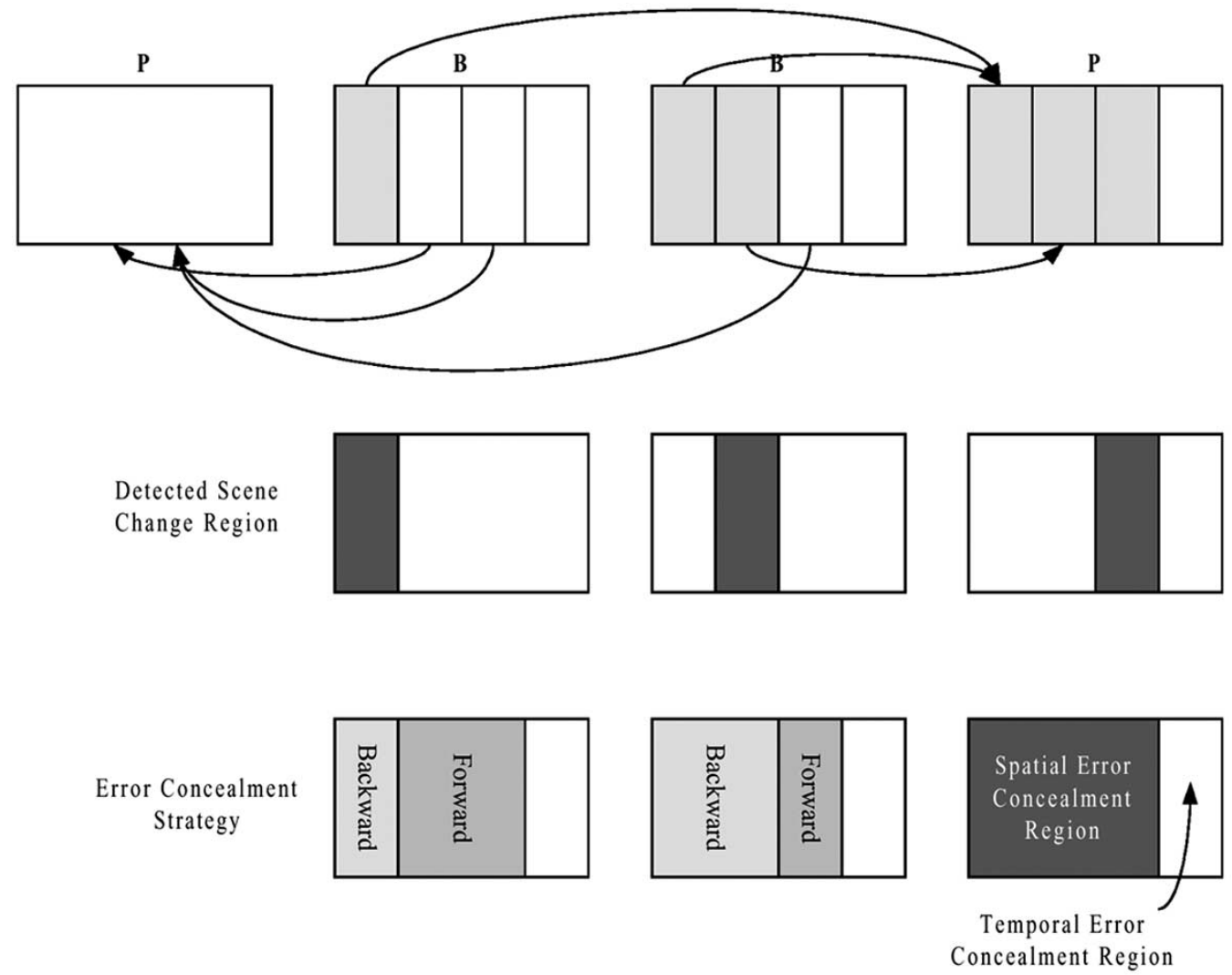

Fig. 15. Illustrations of wipe detection and error concealment strategy.

TABLE IX

Simulation RESUlts OF IMPACT OF FALSE AlARMS

\begin{tabular}{c|c|c}
\hline BER $=2 \%$ false alarm at frame 8 & MCTEC $(\mathrm{dB})$ & Proposed $(\mathrm{dB})$ \\
\hline Miss & 34.70 & 34.56 \\
\hline Sales & 31.56 & 31.10 \\
\hline Pingpong & 30.44 & 30.12 \\
\hline Windmill & 28.46 & 28.02 \\
\hline
\end{tabular}

obvious improvement is obtained. Nevertheless, it is still a very long way to go because the wipe transition is kaleidoscopic just like the richness of daily generation of video.

\section{CONCLUSION}

In this paper, a novel error concealment algorithm is proposed to deal with the error propagation from scene change and the insufficiency of the conventional algorithms. Once the scene change occurs, either abrupt or gradual scene change, the change of the scene contents will cause the conventional temporal error concealment algorithm to replace the corrupt blocks with reference blocks with less or even no temporal correlation. It creates significant concealment error and the error will propagate to all GOP. Our method provides a solution to locate the scene change and adopt corresponding strategies to obtain better concealment results. For abrupt scene change, the nearest $\mathrm{P}$ frame posterior to scene change should be spatial error concealed, and B frames belonging to the same SGOP should be concealed by the anchor frame of the same scene. For gradual scene change, the property of interpolation motivates us to conceal error blocks with interpolation or extrapolation of successful reconstructed block sequence. Simulation results show that significant improvement about 3 to $5 \mathrm{~dB}$ PSNR in average and 6 to even $10 \mathrm{~dB}$ maximum gain is obtained when compared with conventional temporal error concealment algorithm. Last, discussion and simulation also indicate that the influence of the scene change false alarm on the proposed error concealment method is insignificant and overhead computation load and memory space is comparatively small. 


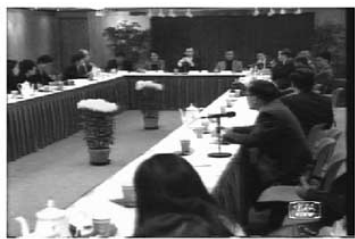

Frame 1

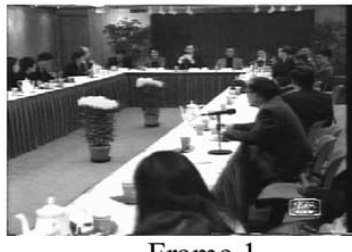

Frame 1

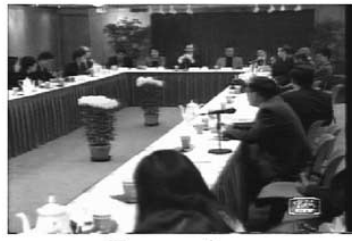

Frame 1

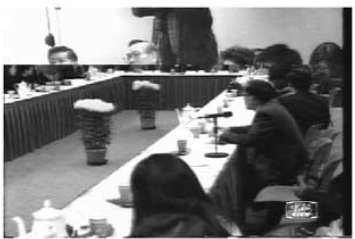

Frame 2

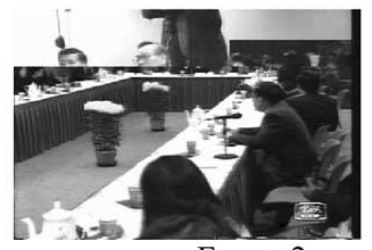

Frame 2

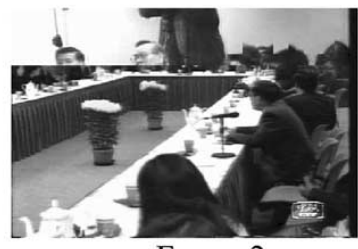

Frame 2

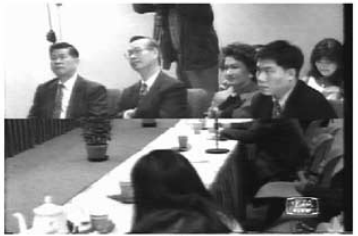

Frame 3

(a)

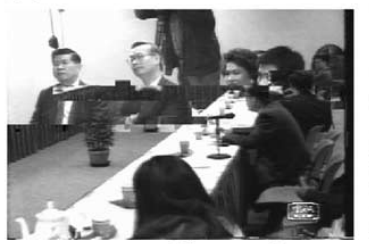

Frame 3

(b)

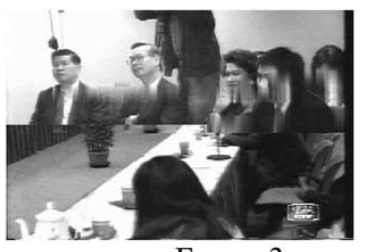

Frame 3

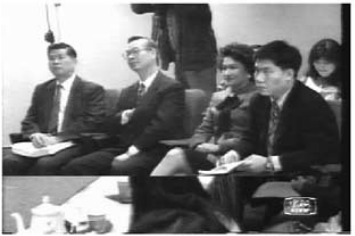

Frame 4

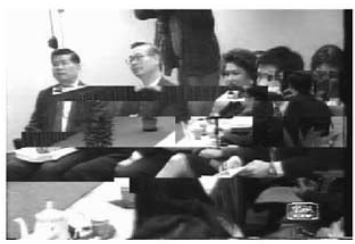

Frame 4

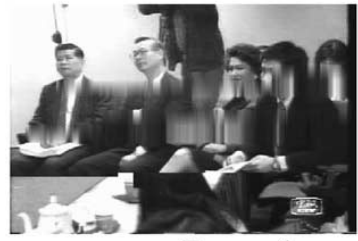

Frame 4

(c)

Fig. 16. Experimental results of error concealment for wipe transitions: (a) original sequences, (b) results of MCTEC, and (c) results of our method.

\section{REFERENCES}

[1] G.-S. Yu, M. M.-K. Liu, and M. W. Marcellin, "POCS-Based error concealment for packet video using multiframe overlap information," IEEE Trans. Circuits Syst. Video Technol., vol. 8, pp. 422-434, Aug. 1998.

[2] Y. J. Chung, J. Kim, and C.-C. J. Kuo, "Real-Time streaming video with adaptive bandwidth control and DCT-based error concealment," IEEE Trans. Circuits Syst. II, vol. 46, pp. 951-956, July 1999.

[3] H. Sun and W. Kwok, "Concealment of damaged block transform coded images using projections onto convex sets," IEEE Trans. Image Processing, vol. 4, pp. 470-477, Apr. 1995.

[4] V. Parthasarathy, J. W. Modestino, and K. S. Vastola, "Design of a transport coding scheme for high-quality video over ATM networks," IEEE Trans. Circuits Syst. Video Technol., vol. 7, pp. 358-376, Apr. 1997.

[5] W. Keck, "A method for robust decoding of erroneous MPEG-2 video bitstreams," IEEE Trans. Consumer Electron., vol. 42, pp. 411-421, Aug. 1996.

[6] J. Feng, K.-T. Lo, and H. Mehrpour, "Error concealment for MPEG video transmissions," IEEE Trans. Consumer Electron., vol. 43, pp. 183-187, May 1997.

[7] W. Zhu, Y. Wang, and Q.-F. Zhu, "Second-Order derivative-based smoothness measure for error concealment in DCT-based codecs," IEEE Trans. Circuits Syst. Video Technol., vol. 8, pp. 713-718, Oct. 1998.

[8] J. F. Arnold, M. R. Frater, and J. Zhang, "Error resilience in the MPEG-2 video coding standard for cell based networks-A review," Signal Process.: Image Commun., vol. 14, pp. 607-633, May 1999.

[9] S. Kaiser and K. Fazel, "Comparison of error concealment techniques for an MPEG-2 video decoder in terrestrial TV-broadcasting," Signal Process.: Image Commun., vol. 14, pp. 655-676, May 1999.

[10] H.-C. Shyu and J.-J. Leou, "Detection and concealment of transmission errors in MPEG-2 images-A genetic algorithm approach," IEEE Trans. Circuits Syst. Video Technol., vol. 9, no. 6, pp. 937-948, Sept. 1999.

[11] J. M. Boyce, "Packet loss resilient transmission of MPEG video over the internet," Signal Process.: Image Commun., vol. 15, pp. 7-24, Sept. 1999.

[12] D. W. Redmill, D. R. Bull, J. T. Chung-How, and N. G. Kingsbury, "Error-resilient image and video coding for wireless communication systems," Electron. Commun. Eng. J., Aug. 1998.

[13] M. Bystrom, V. Parthasarathy, and J. W. Modestino, "Hybrid error concealment schemes for broadcast video transmission over ATM networks," IEEE Trans. Circuits Syst. Video Technol., vol. 9, pp. 868-881, Sept. 1999.
[14] P.-J. Lee and M.-J. Chen, "Robust error concealment algorithm for video decoder," IEEE Trans. Consumer Electron., vol. 45, pp. 851-859, Aug. 1999.

[15] M.-C. Hong, H. Schwab, L. P. Kondi, and A. K. Katsaggelos, "Error concealment algorithms for compressed video," Signal Process.: Image Commun., vol. 14, pp. 473-492, May 1999.

[16] J.-W. Suh and Y.-S. Ho, "Error concealment based on directional interpolation," IEEE Trans. Consumer Electron., vol. 43-3, pp. 295-302, August 1997.

[17] M. Al-Mualla, N. Canagarajah, and D. R. Bull, "Temporal error concealment using motion field interpolation," Electron. Lett., vol. 35-3, pp. 215-217, Feb. 1999.

[18] W.-M. Lam and A. R. Reibman, "An error concealment algorithm for images subject to channel errors," IEEE Trans. Image Process., vol. 4, pp. 533-542, May 1995.

[19] W. Kwok and H. Sun, "Multi-directional interpolation for spatial error concealment," IEEE Trans. Consumer Electron., vol. 39, pp. 455-460, Aug. 1993.

[20] W. Zeng and B. Liu, "Geometric-Structure-Based error concealment with novel applications in block-based low-bit-rate coding," IEEE Trans. Circuits Syst. Video Technol., vol. 9, pp. 648-665, June 1999.

[21] J.-T. Wang and P.-C. Chang, "Error-Propagation prevention technique for real-time video transmission over ATM networks," IEEE Trans. Circuits Syst. Video Technol., vol. 9, pp. 513-523, Apr. 1999.

[22] M.-J. Chen, L.-G. Chen, and R.-M. Weng, "Error concealment of lost motion vectors with overlapped motion compensation," IEEE Trans. Circuits Syst. Video Technol., vol. 7, pp. 560-563, June 1997.

[23] V. Parthasarathy, J. W. Modestino, and K. S. Vastola, "Reliable transmission of high-quality video over ATM networks," IEEE Trans. Image Processing, vol. 8, pp. 361-374, Mar. 1999.

[24] Z. Wang, Y. Yu, and D. Zhang, "Best neighborhood matching: An information loss restoration technique for block-based image coding systems," IEEE Trans. Image Processing, vol. 7, pp. 1056-1061, July 1998.

[25] X. Lee, Y.-Q. Zhang, and A. Leon-Garcia, "Information loss recovery for block-based image coding techniques-a fussy logic approach," IEEE Trans. Image Processing, vol. 4, pp. 259-273, March 1995.

[26] J. W. Park and S. U. Lee, "Recovery of corrupted image data based on the NURBS interpolation," IEEE Trans. Circuits Systems Video Technol., vol. 9, pp. 1003-1008, Oct. 1999.

[27] S.-C. Pei and Y.-Z. Chou, "Efficient MPEG compressed video analysis using macroblock type information," IEEE Trans. Multimedia, vol. 1, pp. 321-333, Dec. 1999. 
[28] J. Meng, Y. Juan, and S.-F. Chang, "Scene change detection in MPEG compressed video sequence," Proc. SPIE, Electron, Imag., vol. 2419 , pp. 14-25, Feb. 1995

[29] S.-C. Pei and Y.-Z. Chou, "Efficient and effective wipe detection in MPEG compressed video based on the macroblock information," in Proc. ICIP, vol. 3, Sept. 2000, pp. 953-956.

[30] A. Nagasaka and Y. Tanaka, "Automatic video indexing and full motion search for object appearances," in Visual Database Systems, II, E. Knuth and L. M. Wegner, Eds. Amsterdam, The Netherlands: North-Holland, 1991, pp. 119-133.

[31] F. Arman, R. Depommier, A. Hsu, and M.-Y. Chiu, "Content-based browsing of video sequences," in ACM Multimedia'94, Aug. 1994, pp. $97-103$.

[32] F. Arman, A. Hsu, and M. Y. Chiu, "Feature management for large video databases," Proc. SPIE, Storage and Retrieval for Image and Video Databases, vol. 1908, pp. 2-12, 1993.

[33] Y. Nakajuma, "A video browsing using fast scene cut detection for an efficient networked video database access," IEICE Trans. Inform. Syst., vol. E77-D, no. 12, Dec. 1994.

[34] F. Arman, A. Hsu, and M. Y. Chiu, "Image processing on compressed data for large video databases," in Proc. First ACM Int. Conf. Multimedia, Aug. 1993, pp. 267-272.

[35] H. J. Zhang, A. Kankanhalli, and S. W. Smoliar, "Automatic partitioning of full-motion video," Multimedia Syst., vol. 1, pp. 10-28, July 1993.

[36] H. J. Zhang, C. Y. Low, and S. W. Smoliar, "Video parsing and browsing using compressed data," Multimedia Tools Applicat., vol. 1, no. 1, pp. 89-111, Mar. 1995.

[37] B.-L. Yeo and B. Liu, "Rapid scene analysis on compressed video," IEEE Trans. Circuits Systems Video Technol., vol. 5, pp. 533-544, Dec. 1995.

[38] K. Otsuji and Y. Tonomura, "Projection detecting filter for video cut detection," in Proc. First ACM Int. Conf. Multimedia, Aug. 1993, pp. 251-257.

[39] S. F. Chang and D. G. Messerschmitt, "Manipulation and compositing of MC-DCT compressed video," IEEE J. Select. Areas Commun., vol. 13, pp. 1-11, Jan. 1995

[40] Patel and Sethi, "Compressed video processing for cut detection," Proc. Inst. Elect. Eng., Vis., Image, Signal Process., Oct. 1996.

[41] _ " "Video shot detection and characterization for video databases," Pattern Recognit., vol. 30, no. 4, pp. 583-592, Mar. 1997.

[42] Yeo and Liu, "Visual content highlighting via automatic extraction of embedded captions on MPEG compressed video," Proc. SPIE, Digital Video Compression: Alg. Technol., vol. 2668, pp. 58-47, 1996.

[43] Meng, Juan, and Chang, "Scene change detection in a MPEG compressed video sequence," Proc. SPIE, Digital Video Compression: Alg. Technol., vol. 2419, pp. 14-25, 1995.
[44] Wei, Zhang, and Zhong, "A robust approach to video segmentation using compressed data," Proc. SPIE, Storage and Retrieval for Still Image and Video Database, vol. 3022, pp. 448-456, 1997.

[45] R. M. Ford, C. Robson, D. Temple, and M. Gerlach, "Metrics for shot boundary detection in digital video sequences," Multimedia Syst., vol. 8 , no. 1 , pp. 37-46, 2000

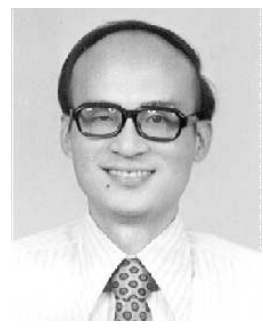

Soo-Chang Pei (S'71-M'86-SM'89-F'00) was born in Soo-Auo, Taiwan, R.O.C., in 1949. He received the B.S.E.E. degree from National Taiwan University (NTU), Taipei, in 1970 and M.S.E.E. and $\mathrm{Ph} . \mathrm{D}$. degrees from the University of California, Santa Barbara (UCSB), in 1972 to 1975 , respectively.

He was an Engineering Officer in the Chinese Navy Shipyard from 1970 to 1971. From 1971 to 1975 , he was a Research Assistant at UCSB. $\mathrm{He}$ was Professor and Chairman in the Electrical Engineering Department, Tatung Institute of Technology and NTU from 1981 to 1983 and 1995 to 1998 , respectively. Presently, he is Professor, Electrical Engineering Department, NTU. His research interests include digital signal processing, image processing, optical information processing, and laser holography.

Dr. Pei received the National Sun Yat-Sen Academic Achievement Award in Engineering in 1984, the Distinguished Research Award from the National Science Council from 1990 to 1998, Outstanding Electrical Engineering Professor Award from the Chinese Institute of Electrical Engineering in 1998, and the Academic Achievement Award in Engineering from the Ministry of Education in 1998. He was President of the Chinese Image Processing and Pattern Recognition Society of Taiwan from 1996 to 1998 and is a member of Eta Kappa Nu and the Optical Society of America.

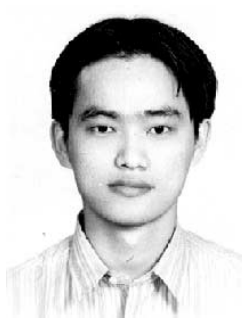

Yu-Zuong Chou was born in Tainan, Taiwan, R.O.C He received the B.S. degree from the National Tsing Hua University, Hsinchu, Taiwan, in 1994, and the M.S. and Ph.D. degrees from the National Taiwan University (NTU), Taipei, in 1996 and 2002, respectively, both in electrical engineering.

He is currently an Engineer with Realtek Semiconductor Corporation, Hsinchu. His current research interests include video compression, image processing, multimedia application and SOC design. 